\title{
Simultaneous determination of CKM angle $\gamma$ and charm mixing parameters
}

\section{LHCb}

\section{The LHCb collaboration}

E-mail: matthew.william.kenzie@cern.ch

ABSTRACT: A combination of measurements sensitive to the $C P$ violation angle $\gamma$ of the Cabibbo-Kobayashi-Maskawa unitarity triangle and to the charm mixing parameters that describe oscillations between $D^{0}$ and $\bar{D}^{0}$ mesons is performed. Results from the charm and beauty sectors, based on data collected with the LHCb detector at CERN's Large Hadron Collider, are combined for the first time. This method provides an improvement on the precision of the charm mixing parameter $y$ by a factor of two with respect to the current world average. The charm mixing parameters are determined to be $x=\left(0.400_{-0.053}^{+0.052}\right) \%$ and $y=\left(0.630_{-0.030}^{+0.033}\right) \%$. The angle $\gamma$ is found to be $\gamma=\left(65.4_{-4.2}^{+3.8}\right)^{\circ}$ and is the most precise determination from a single experiment.

KeYwords: $B$ physics, Charm physics, CKM angle gamma, CP violation, Hadron-Hadron scattering (experiments)

ArXiv EPrint: 2110.02350 


\section{Contents}

$\begin{array}{llr}1 & \text { Introduction } & 1\end{array}$

2 Assumptions $\quad 6$

$\begin{array}{llr}3 & \text { Statistical treatment } & 7\end{array}$

4 Results $\quad 8$

5 Conclusion 13

$\begin{array}{ll}\text { A Correlation matrix } & 14\end{array}$

$\begin{array}{ll}\text { B Contribution of each input measurement to the global } \chi^{2} & 17\end{array}$

$\begin{array}{ll}\text { C Pull distribution of each input observable } & 17\end{array}$

$\begin{array}{ll}\text { D Additional figures } & 21\end{array}$

$\begin{array}{lr}\text { The LHCb collaboration } & 26\end{array}$

\section{Introduction}

Precise measurements of the Cabibbo-Kobayashi-Maskawa (CKM) unitarity triangle provide a strict test of the Standard Model (SM) and allow for indirect new physics searches in the quark sector up to very high mass scales. The $C P$ violating phase $\gamma \equiv \arg \left[-V_{u d} V_{u b}^{*} / V_{c d} V_{c b}^{*}\right]$, where $V_{q q^{\prime}}$ is the relevant CKM matrix element, is the only angle of the unitarity triangle that can be determined using solely measurements of treelevel $B$-meson decays [1-8] with negligible theoretical uncertainty [9], assuming no sizeable new physics effects are present at tree level [10]. Deviations between direct measurements of $\gamma$ and the value derived from global CKM fits, which assume validity of the SM and hence unitarity of the CKM matrix, would be a clear indication of physics beyond the SM. Furthermore, comparisons between the value of $\gamma$ measured using decays of different $B$-meson species provide sensitivity to possible new physics effects at tree level given the different decay topologies involved. The world average for direct measurements of $\gamma=\left(66.2_{-3.6}^{+3.4}\right)^{\circ}[11]$ is dominated by LHCb results. The experimental uncertainty on $\gamma$ is larger than that obtained from global CKM fits, $\gamma=\left(65.6_{-2.7}^{+0.9}\right)^{\circ}[12]$ using a frequentist framework, and $\gamma=(65.8 \pm 2.2)^{\circ}$ [13] with a Bayesian approach. Closing this sensitivity gap is a key physics goal of the LHCb experiment and the comparison between the direct and indirect determinations of $\gamma$ is an important test of the SM. 

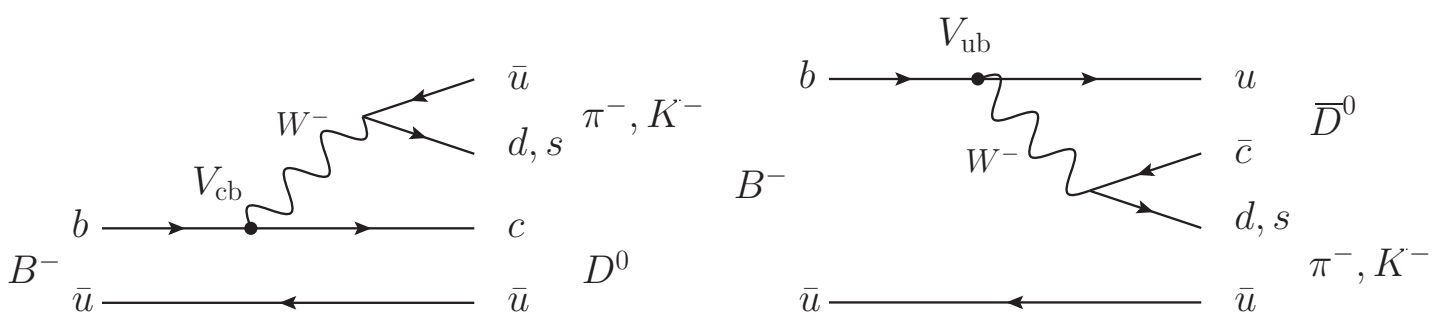

Figure 1. Leading-order Feynman diagrams for $B^{-} \rightarrow D h^{-}$decays with a (left) favoured $b \rightarrow c$ and (right) suppressed $b \rightarrow u$ quark transition.

The CKM angle $\gamma$ is measured in decays which are sensitive to interference between favoured $b \rightarrow c$ and suppressed $b \rightarrow u$ quark transition amplitudes that are proportional to $V_{c b}$ and $V_{u b}$, respectively. ${ }^{1}$ The ratio of these two amplitudes is given by $\mathcal{A}_{\text {sup }} / \mathcal{A}_{\text {fav }}=r_{B} e^{i \delta_{B} \pm \gamma}$, where the + or - sign indicates whether the initial state contains a $\bar{b}$ - or $b$-quark, $r_{B}$ is the ratio of the amplitude magnitudes, and $\delta_{B}$ their $C P$-conserving strong-phase difference. This interference effect is typically measured in $B$-meson decays such as $B^{ \pm} \rightarrow D h^{ \pm}$, where $D$ is an admixture of the $D^{0}$ and $\bar{D}^{0}$ flavour states, and $h^{ \pm}$ is either a charged kaon or pion. Figure 1 shows the leading-order Feynman diagrams for the favoured and suppressed processes. Interference effects, providing sensitivity to $\gamma$, only occur when the $D$ meson decays to a final state, $f$, accessible to both $D^{0}$ and $\bar{D}^{0}$ mesons. Neglecting mixing in the neutral charm system, the decay rate for a $B^{ \pm} \rightarrow D h^{ \pm}$decay is given by

$$
\Gamma\left(B^{ \pm} \rightarrow D h^{ \pm}\right) \propto\left|r_{D} e^{-i \delta_{D}}+r_{B} e^{i\left(\delta_{B} \pm \gamma\right)}\right|^{2} \Rightarrow r_{D}^{2}+r_{B}^{2}+2 \kappa_{D} \kappa_{B} r_{D} r_{B} \cos \left(\delta_{B}+\delta_{D} \pm \gamma\right),
$$

where $r_{D}$ and $\delta_{D}$ are the magnitude ratio and strong-phase difference between the $D^{0} \rightarrow f$ and $\bar{D}^{0} \rightarrow f$ amplitudes. For $D$ decays to $C P$ eigenstates, e.g. $D \rightarrow K^{+} K^{-}$, these values are $r_{D}=1$ and $\delta_{D}=0$. The coherence factors of $B$ and $D$ decays, $\kappa_{B}$ and $\kappa_{D}$, are equal to unity for two-body decays, and account for a dilution of the interference term due to incoherence (strong phase variation) between contributing intermediate resonances in multibody decays. The hadronic parameters, $r_{B}, \delta_{B}, r_{D}, \delta_{D}$, are specific to each $B$ decay and subsequent $D$ decay, respectively. However, the $C P$-violating weak phase difference between $B^{+}$and $B^{-}$amplitudes, $\gamma$, is shared by all such decays.

Equation (1.1) has at least five unknown parameters, even more if the coherence factors are not set to unity, hence they cannot be determined using a single pair of $B^{ \pm}$decay rates. This is overcome by combining the results from many different $D$-decay modes to overconstrain the parameters of the $B$-meson decay, provided that the corresponding $r_{D}$, $\delta_{D}$ and $\kappa_{D}$ parameters are constrained by other measurements. In past combinations these parameters have been taken as external inputs using dedicated charm-meson measurements. The large $B$-meson samples now constrain $\gamma$ and $\delta_{B}$ so precisely that $\delta_{D}^{K \pi}$, the strong phase difference between $D^{0} \rightarrow K^{-} \pi^{+}$and $\bar{D}^{0} \rightarrow K^{-} \pi^{+}$decays, can be measured with similar precision as $\gamma$ and $\delta_{B}$, a factor of about two better than the previous world average [11].

\footnotetext{
${ }^{1}$ Charge conjugation is implied throughout unless stated otherwise.
} 
This improved precision on $\delta_{D}^{K \pi}$ can then be used to improve knowledge of charm mixing as described below.

The mass eigenstates of the neutral charm mesons can be written as $\left|D_{1,2}\right\rangle \equiv p\left|D^{0}\right\rangle \pm q\left|\bar{D}^{0}\right\rangle$, where $p$ and $q$ are complex parameters such that $|p|^{2}+|q|^{2}=1$. The $D_{1}\left(D_{2}\right)$ state corresponds to the $+(-)$ sign and is approximately $C P$ even (odd) in the chosen convention. The mixing of charm flavour states can be described by two dimensionless parameters, $x \equiv\left(m_{1}-m_{2}\right) / \Gamma$ and $y \equiv\left(\Gamma_{1}-\Gamma_{2}\right) / 2 \Gamma$, where $m_{i}\left(\Gamma_{i}\right)$ is the mass (width) of the appropriate $D$ mass state, and $\Gamma$ their average decay width. ${ }^{2}$ Effects of $C P$ violation in $D^{0}$ and $\bar{D}^{0}$ decays to a common final state, $f$, can be seen in mixing if $|q / p| \neq 1$, or in the interference between mixing and decay if $\phi \equiv \arg (q / p) \neq 0, \pi \cdot{ }^{3}$ Study of the charm mixing parameters is of high interest in its own right, because the flavourchanging neutral currents responsible for the mixing transition do not occur at tree-level in the SM, and thus can be significantly affected by contributions from new heavy particles. The world averages for $x=\left(4.09_{-0.49}^{+0.48}\right) \times 10^{-3}$ and $y=\left(6.15_{-0.55}^{+0.56}\right) \times 10^{-3}$ [11] are dominated by $\mathrm{LHCb}$ results.

The mixing parameters, $x$ and $y$, can be determined using the ratio of wrong-sign (WS), $D^{0} \rightarrow K^{+} \pi^{-}$, and right-sign (RS), $D^{0} \rightarrow K^{-} \pi^{+}$, time-dependent decay rates. This ratio is

$$
R^{ \pm}(t) \approx R^{ \pm}+\sqrt{R^{ \pm}} y^{\prime \pm}\left(\frac{t}{\tau}\right)+\frac{\left(x^{ \pm}\right)^{2}+\left(y^{\prime \pm}\right)^{2}}{4}\left(\frac{t}{\tau}\right)^{2}
$$

up to second order in the mixing parameters, where $t$ is the decay time, $\tau$ is the $D^{0}$ meson lifetime, and the $+(-)$ signs correspond to the decay-rate ratio for a flavour-tagged $D^{0}\left(\bar{D}^{0}\right)$ initial state. ${ }^{4}$ The parameter $R^{ \pm}=r_{D}^{2}\left(1 \pm A_{D}\right)$ is the ratio of suppressed-tofavoured decay rates, modulated by the direct $C P$ asymmetry, $A_{D}$, between $D^{0}$ and $\bar{D}^{0}$ WS decays. The parameters $x^{\prime \pm} \equiv-|q / p|^{ \pm 1}\left[x \cos \left(\delta_{D}^{K \pi} \pm \phi\right)+y \sin \left(\delta_{D}^{K \pi} \pm \phi\right)\right]$ and $y^{\prime \pm} \equiv-|q / p|^{ \pm 1}\left[y \cos \left(\delta_{D}^{K \pi} \pm \phi\right)-x \sin \left(\delta_{D}^{K \pi} \pm \phi\right)\right]$ encode the mixing. Since $\delta_{D}^{K \pi}$ is close to $\pi$ and $\phi$ is almost zero [11], it follows that $R^{ \pm}(t)$ is mostly sensitive to the parameter $y$ through the term linear in decay time and mixing parameters, and currently the precision on $y$ is limited by the precision with which $\delta_{D}^{K \pi}$ is known. Consequently, a simultaneous combination using both beauty and charm observables from $\mathrm{LHCb}$ is performed for the first time, improving the precision on $y(x)$ by about $50 \%(2 \%)$.

A further motivation for the simultaneous combination of both beauty and charm measurements is that non-negligible effects due to charm-meson mixing give rise to additional terms in eq. (1.1). Incorporating the effect of $D$-meson mixing, up to first order in $x$ and

\footnotetext{
${ }^{2}$ Natural units, with $c=\hbar=1$, are used throughout.

${ }^{3}$ The Wolfenstein parametrisation and the convention that $C P\left|D^{0}\right\rangle=\left|\bar{D}^{0}\right\rangle$ is used.

${ }^{4}$ It should be noted that there are multiple conventions in the literature for the strong phase $\delta_{D}^{K \pi}$, depending on whether the discussion involves the CKM angle $\gamma$ or charm mixing. The convention in which $\delta_{D} \rightarrow \pi$ in the $\mathrm{SU}(3)$ limit is used, which is shifted by $\pi$ with respect to the convention employed by the HFLAV Charm group.
} 
$y$, means the decay rate of eq. (1.1) becomes [14]

$$
\begin{aligned}
\Gamma\left(B^{ \pm} \rightarrow D h^{ \pm}\right) \propto & r_{D}^{2}+r_{B}^{2}+2 \kappa_{D} \kappa_{B} r_{D} r_{B} \cos \left(\delta_{B}+\delta_{D} \pm \gamma\right) \\
& -\alpha\left[\left(1+r_{B}^{2}\right) \kappa_{D} r_{D} \cos \left(\delta_{D}\right)+\left(1+r_{D}^{2}\right) \kappa_{B} r_{B} \cos \left(\delta_{B} \pm \gamma\right)\right] y \\
& +\alpha\left[\left(1-r_{B}^{2}\right) \kappa_{D} r_{D} \sin \left(\delta_{D}\right)-\left(1-r_{D}^{2}\right) \kappa_{B} r_{B} \sin \left(\delta_{B} \pm \gamma\right)\right] x
\end{aligned}
$$

where the $\alpha$ coefficient accounts for the non-uniform decay-time acceptance of the LHCb detector. For cases where $r_{B} \gg x, y$, such as the $B^{ \pm} \rightarrow D K^{ \pm}$decay, the effect of $D$ mixing is small. However, for decays like $B^{ \pm} \rightarrow D \pi^{ \pm}$, where $r_{B} \sim x, y$, the effect is significant [14]. Studies of this combination, which includes both $B^{ \pm} \rightarrow D K^{ \pm}$and $B^{ \pm} \rightarrow D \pi^{ \pm}$modes, suggest that not accounting for the effect of $D$-meson mixing results in a bias on $\gamma$ of approximately $1.8^{\circ}$, and an ever larger bias for the hadronic parameters, $r_{B^{ \pm}}^{D \pi^{ \pm}}$and $\delta_{B^{ \pm}}^{D \pi^{ \pm}}$, of the $B^{ \pm} \rightarrow D \pi^{ \pm}$system. Thus an unbiased determination of $\gamma, x$ and $y$ requires the simultaneous combination produced in this article.

This article presents results for the weak phase $\gamma$ and charm mixing and $C P$ violation parameters $x, y,|q / p|$ and $\phi$, as well as for several additional amplitude ratios and strong phases, using data collected at the LHCb experiment during the first two runs of the LHC. The statistical procedure is identical to that described in ref. [15] and follows a frequentist treatment which is described in detail in ref. [16] and briefly recapped in section 3 . The results have additionally been cross-checked using Bayesian inference, which finds very similar values. The results presented here supersede previous LHCb combinations [15-18].

The full list of $\mathrm{LHCb}$ measurements that are used as inputs to the combination is provided in table 1 . In the beauty sector this includes decay-rate ratios and charge asymmetries of $B^{ \pm} \rightarrow D h^{ \pm}, B^{ \pm} \rightarrow D^{*} h^{ \pm}, B^{ \pm} \rightarrow D K^{* \pm}, B^{ \pm} \rightarrow D h^{ \pm} \pi^{+} \pi^{-}, B^{0} \rightarrow D K^{* 0}$, $B^{0} \rightarrow D^{\mp} \pi^{ \pm}, B_{s}^{0} \rightarrow D_{s}^{\mp} K^{ \pm}$and $B_{s}^{0} \rightarrow D_{s}^{\mp} K^{ \pm} \pi^{+} \pi^{-}$decays, where $D^{*}$ is an admixture of $D^{* 0}$ and $\bar{D}^{* 0}$ flavour states. In the charm sector this includes time-dependent measurements of $D^{0} \rightarrow h^{+} h^{-}, D^{0} \rightarrow K^{+} \pi^{-}, D^{0} \rightarrow K^{ \pm} \pi^{\mp} \pi^{+} \pi^{-}$and $D^{0} \rightarrow K_{\mathrm{S}}^{0} \pi^{+} \pi^{-}$decays. There are seven new or updated measurements from beauty-meson decays since the last combination, including LHCb Run 2 updates from the highly sensitive $B^{ \pm} \rightarrow D h^{ \pm}$with $D \rightarrow K_{\mathrm{S}}^{0} h^{+} h^{-}$[19] and $D \rightarrow h^{+} h^{-}$[20] decays. The eight inputs from LHCb charm analyses are included in the combination for the first time.

Additional external constraints are summarised in table 2; these are used predominantly to provide auxiliary information on the hadronic parameters and coherence factors in multibody $B$ and $D$ decays. In the case of quasi- $C P$-eigenstate decays, such as $D \rightarrow \pi^{+} \pi^{-} \pi^{+} \pi^{-}$, the coherence factor is determined by the fraction of $C P$-even content in the final-state amplitude, $F^{+}=\left(\kappa_{D}+1\right) / 2$. In the case of the $B^{0} \rightarrow D^{\mp} \pi^{ \pm}, B_{s}^{0} \rightarrow D_{s}^{\mp} K^{ \pm}$ and $B_{s}^{0} \rightarrow D_{s}^{\mp} K^{ \pm} \pi^{+} \pi^{-}$modes, the weak phases measured through the time-dependent $C P$ asymmetry are $(\gamma+2 \beta)$ and $\left(\gamma-2 \beta_{s}\right)$, induced via interference between $B_{(s)}^{0}$ mixing and decay. Therefore, in order to obtain sensitivity to $\gamma$, external constraints from the world averages of $\beta \equiv \arg \left[-V_{c d} V_{c b}^{*} / V_{t d} V_{t b}^{*}\right]$ and $\phi_{s} \approx-2 \beta_{s} \equiv-2 \arg \left[-V_{t s} V_{t b}^{*} / V_{c s} V_{c b}^{*}\right]$ [11] are included. 


\begin{tabular}{|c|c|c|c|c|}
\hline$B$ decay & $D$ decay & Ref. & Dataset & $\begin{array}{l}\text { Status since } \\
\text { Ref. [17] }\end{array}$ \\
\hline$B^{ \pm} \rightarrow D h^{ \pm}$ & $D \rightarrow h^{+} h^{-}$ & {$[20]$} & Run $1 \& 2$ & Updated \\
\hline$B^{ \pm} \rightarrow D h^{ \pm}$ & $D \rightarrow h^{+} \pi^{-} \pi^{+} \pi^{-}$ & {$[21]$} & Run 1 & As before \\
\hline$B^{ \pm} \rightarrow D h^{ \pm}$ & $D \rightarrow h^{+} h^{-} \pi^{0}$ & {$[22]$} & Run 1 & As before \\
\hline$B^{ \pm} \rightarrow D h^{ \pm}$ & $D \rightarrow K_{\mathrm{S}}^{0} h^{+} h^{-}$ & {$[19]$} & Run $1 \& 2$ & Updated \\
\hline$B^{ \pm} \rightarrow D h^{ \pm}$ & $D \rightarrow K_{\mathrm{S}}^{0} K^{ \pm} \pi^{\mp}$ & {$[23]$} & Run $1 \& 2$ & Updated \\
\hline$B^{ \pm} \rightarrow D^{*} h^{ \pm}$ & $D \rightarrow h^{+} h^{-}$ & {$[20]$} & Run $1 \& 2$ & Updated \\
\hline$B^{ \pm} \rightarrow D K^{* \pm}$ & $D \rightarrow h^{+} h^{-}$ & {$[24]$} & Run $1 \& 2(*)$ & As before \\
\hline$B^{ \pm} \rightarrow D K^{* \pm}$ & $D \rightarrow h^{+} \pi^{-} \pi^{+} \pi^{-}$ & {$[24]$} & Run $1 \& 2\left(^{*}\right)$ & As before \\
\hline$B^{ \pm} \rightarrow D h^{ \pm} \pi^{+} \pi^{-}$ & $D \rightarrow h^{+} h^{-}$ & {$[25]$} & Run 1 & As before \\
\hline$B^{0} \rightarrow D K^{* 0}$ & $D \rightarrow h^{+} h^{-}$ & {$[26]$} & Run $1 \& 2(*)$ & Updated \\
\hline$B^{0} \rightarrow D K^{* 0}$ & $D \rightarrow h^{+} \pi^{-} \pi^{+} \pi^{-}$ & {$[26]$} & Run $1 \& 2(*)$ & New \\
\hline$B^{0} \rightarrow D K^{* 0}$ & $D \rightarrow K_{\mathrm{S}}^{0} \pi^{+} \pi^{-}$ & {$[27]$} & Run 1 & As before \\
\hline$B^{0} \rightarrow D^{\mp} \pi^{ \pm}$ & $D^{+} \rightarrow K^{-} \pi^{+} \pi^{+}$ & {$[28]$} & Run 1 & As before \\
\hline$B_{s}^{0} \rightarrow D_{s}^{\mp} K^{ \pm}$ & $D_{s}^{+} \rightarrow h^{+} h^{-} \pi^{+}$ & {$[29]$} & Run 1 & As before \\
\hline$B_{s}^{0} \rightarrow D_{s}^{\mp} K^{ \pm} \pi^{+} \pi^{-}$ & $D_{s}^{+} \rightarrow h^{+} h^{-} \pi^{+}$ & {$[30]$} & Run $1 \& 2$ & New \\
\hline$D$ decay & Observable(s) & Ref. & Dataset & $\begin{array}{l}\text { Status since } \\
\text { Ref. }[17]\end{array}$ \\
\hline$D^{0} \rightarrow h^{+} h^{-}$ & $\Delta A_{C P}$ & {$[31-33]$} & Run $1 \& 2$ & New \\
\hline$D^{0} \rightarrow h^{+} h^{-}$ & $y_{C P}$ & {$[34]$} & Run 1 & New \\
\hline$D^{0} \rightarrow h^{+} h^{-}$ & $\Delta Y$ & {$[35-38]$} & Run $1 \& 2$ & New \\
\hline$D^{0} \rightarrow K^{+} \pi^{-}$(Single Tag) & $R^{ \pm},\left(x^{\prime \pm}\right)^{2}, y^{\prime \pm}$ & {$[39]$} & Run 1 & New \\
\hline$D^{0} \rightarrow K^{+} \pi^{-}$(Double Tag) & $R^{ \pm},\left(x^{ \pm}\right)^{2}, y^{\prime \pm}$ & {$[40]$} & Run $1 \& 2(*)$ & New \\
\hline$D^{0} \rightarrow K^{ \pm} \pi^{\mp} \pi^{+} \pi^{-}$ & $\left(x^{2}+y^{2}\right) / 4$ & {$[41]$} & Run 1 & New \\
\hline$D^{0} \rightarrow K_{\mathrm{S}}^{0} \pi^{+} \pi^{-}$ & $x, y$ & {$[42]$} & Run 1 & New \\
\hline$D^{0} \rightarrow K_{\mathrm{S}}^{0} \pi^{+} \pi^{-}$ & $x_{C P}, y_{C P}, \Delta x, \Delta y$ & {$[43]$} & Run 1 & New \\
\hline$D^{0} \rightarrow K_{\mathrm{S}}^{0} \pi^{+} \pi^{-}$ & $x_{C P}, y_{C P}, \Delta x, \Delta y$ & {$[44]$} & Run 2 & New \\
\hline
\end{tabular}

Table 1. Measurements used in the combination. Inputs from the charm system appear in the lower part of the table. Those that are new, or that have changed, since the previous combination [17] are highlighted in bold. Measurements denoted by $\left(^{*}\right)$ include only a fraction of the Run 2 sample, corresponding to data taken in 2015 and 2016. Where multiple references are cited, measured values are taken from the most recent results, which include information from the others. 


\begin{tabular}{lllll}
\hline Decay & Parameters & Source & Ref. & $\begin{array}{l}\text { Status since } \\
\text { Ref. [17] }\end{array}$ \\
\hline$B^{ \pm} \rightarrow D K^{* \pm}$ & $\kappa_{B^{ \pm}}^{D K^{* \pm}}$ & LHCb & {$[24]$} & As before \\
$B^{0} \rightarrow D K^{* 0}$ & $\kappa_{B^{0}}^{D K^{* 0}}$ & LHCb & {$[45]$} & As before \\
$B^{0} \rightarrow D^{\mp} \pi^{ \pm}$ & $\beta$ & HFLAV & {$[11]$} & Updated \\
$B_{s}^{0} \rightarrow D_{s}^{\mp} K^{ \pm}(\pi \pi)$ & $\phi_{s}$ & HFLAV & {$[11]$} & Updated \\
$D \rightarrow h^{+} h^{-} \pi^{0}$ & $F_{\pi \pi \pi^{0}}^{+}, F_{K \pi \pi^{0}}^{+}$ & CLEO-c & {$[46]$} & As before \\
$D \rightarrow \pi^{+} \pi^{-} \pi^{+} \pi^{-}$ & $F_{4 \pi}^{+}$ & CLEO-c & {$[46]$} & As before \\
$D \rightarrow K^{+} \pi^{-} \pi^{0}$ & $r_{D}^{K \pi \pi^{0}}, \delta_{D}^{K \pi \pi^{0}}, \kappa_{D}^{K \pi \pi^{0}}$ & CLEO-c+LHCb+BESIII & {$[47-49]$} & Updated \\
$D \rightarrow K^{ \pm} \pi^{\mp} \pi^{+} \pi^{-}$ & $r_{D}^{K 3 \pi}, \delta_{D}^{K 3 \pi}, \kappa_{D}^{K 3 \pi}$ & CLEO-c+LHCb+BESIII & {$[41,47-49]$} & Updated \\
$D \rightarrow K_{\mathrm{S}}^{0} K^{ \pm} \pi^{\mp}$ & $r_{D}^{K_{\mathrm{S}}^{0} K \pi}, \delta_{D}^{K_{\mathrm{S}}^{0} K \pi}, \kappa_{D}^{K_{\mathrm{S}}^{0} K \pi}$ & CLEO & {$[50]$} & As before \\
$D \rightarrow K_{\mathrm{S}}^{0} K^{ \pm} \pi^{\mp}$ & $r_{D}^{K_{\mathrm{S}}^{0} K \pi}$ & LHCb & {$[51]$} & As before \\
\hline
\end{tabular}

Table 2. Auxiliary inputs used in the combination. Those highlighted in bold have changed since the previous combination [17].

\section{Assumptions}

The mathematical formulae relating the input observables to the parameters of interest, via eq. (1.3), contain a few assumptions. These are detailed below and their impact on the results has been checked to be negligible at the current precision. In the future, as the precision on $\gamma$ approaches one degree, many of them will need to be reassessed.

Neutral kaon mixing. The extraction of $\gamma$ from decays where the final state of the $D$ meson decay contains a neutral kaon is affected by $C P$ violation in $K^{0}-\bar{K}^{0}$ mixing and decay and by regeneration [52]. For the $D \rightarrow K_{\mathrm{S}}^{0} h^{+} h^{-}$final state, a relative shift of approximately $\Delta \gamma / \gamma \approx \mathcal{O}\left(10^{-3}\right)$ is expected; this has been studied in detail in ref. [52]. Furthermore, the result of the relevant input analysis includes a small systematic uncertainty to account for this [19], so these effects are not considered further in this combination. The size of the effect in $D \rightarrow K_{\mathrm{S}}^{0} K^{ \pm} \pi^{\mp}$ final states is larger, $\Delta \gamma / \gamma \approx \mathcal{O}\left(\varepsilon_{K} / r_{B}\right)$, where $\varepsilon_{K}=\left(2.10_{-0.20}^{+0.27}\right) \times 10^{-3}$ quantifies $C P$ violation in neutral kaon mixing [12]. However, the impact on $\gamma$ is negligible at present because the sensitivity of the input measurement is relatively low [23].

$\boldsymbol{C P}$ violation in $\boldsymbol{D}$-meson decays. The effect of $C P$ violation in the direct decay of $D^{0} \rightarrow K_{\mathrm{S}}^{0} h^{+} h^{-}$is not considered because it is negligible in the SM for the Cabibbofavoured $(\mathrm{CF})$ and doubly Cabibbo-suppressed (DCS) amplitudes contributing to that process. However, the effect of $C P$ violation in the direct decay of $D^{0} \rightarrow K^{+} \pi^{-}$is allowed for in the charm part of the combination, and is denoted by $A_{D}$. A non-zero value of $A_{D}$ would cause a small shift in the charge asymmetries measured for $D^{0} \rightarrow K^{+} \pi^{-}$final states in the beauty system, which is not accounted for in this combination. The impact on the determination of $\gamma$ is found to be smaller than $0.2^{\circ}$. The difference between the size of direct $C P$ violation in $D^{0} \rightarrow K^{+} K^{-}$and $D^{0} \rightarrow \pi^{+} \pi^{-}$decays is included as an input 
in the charm part of the fit [31]. In the beauty system, this value is used to account for direct $C P$ violation in $D^{0} \rightarrow h^{+} h^{-}$decays for the most sensitive analyses, where the $D$ meson is produced in $B^{ \pm} \rightarrow D h^{ \pm}$or $B^{ \pm} \rightarrow D^{*} h^{ \pm}$decays, under the hypothesis of $U$-spin symmetry, $A_{C P}(K K)=-A_{C P}(\pi \pi)=\Delta A_{C P} / 2$, where $A_{C P}(f)$ is the $C P$ asymmetry of the $D$ meson decay to the final state $f$. Any $U$-spin breaking effects are negligible given that ignoring any direct $C P$ violation in $D \rightarrow h^{+} h^{-}$decays only has a small impact, below $0.3^{\circ}$, on the determination of $\gamma$. Time-dependent $C P$ violation in charm mixing, which would add additional terms to eq. (1.3), is also neglected in the beauty system, since its impact on the determination of $\gamma$ is smaller than $0.1^{\circ}[14]$.

Strong phases in $\boldsymbol{D} \rightarrow \boldsymbol{K}_{\mathrm{S}}^{0} \boldsymbol{h}^{+} \boldsymbol{h}^{-}$decays. The input measurements containing $D \rightarrow K_{\mathrm{S}}^{0} h^{+} h^{-}$final states $[19,27,43,44]$, both in the charm and beauty systems, require external knowledge of the strong-phase difference between the $D^{0} \rightarrow K_{\mathrm{S}}^{0} h^{+} h^{-}$and $\bar{D}^{0} \rightarrow K_{\mathrm{S}}^{0} h^{+} h^{-}$amplitudes across the phase space of the $D$ decay. These values are taken from a combination of CLEO-c and BES-III measurements [53-56] and their uncertainties propagated to the uncertainties of the input measurements listed in table 1 . In this combination, each set of input measurements is treated as statistically independent and thus a small part of the already sub-dominant systematic uncertainties of these measurements is being counted twice in the $D \rightarrow K_{\mathrm{S}}^{0} \pi^{+} \pi^{-}$system i.e. the appropriate correlation is not accounted for. This correlation is non-trivial to compute owing to the different binning schemes employed by the different input analyses. In any case, the effect on this combination is small since the uncertainty on the strong phases accounts for approximately $0.5^{\circ}$ of the uncertainty on $\gamma, 40 \%$ of the uncertainty on $x$ and $1 \%$ of the uncertainty on $y$, and these parameters are nearly uncorrelated. Studies suggest that incorporation of this correlation will become important only with a three times larger data sample.

Correlations of systematic uncertainties between input measurements. In addition to the effect of strong phases in $D \rightarrow K_{\mathrm{S}}^{0} h^{+} h^{-}$decays, there are various other potential systematic correlations that are not accounted for. Whilst the individual input analyses provide both statistical and systematic covariance matrices between the sets of observables they measure, there are in principle sub-leading systematic correlations between input analyses which are not accounted for. For example, systematic uncertainties originating from production and detection asymmetries will be correlated for most timeintegrated measurements and those originating from knowledge of decay-time acceptance and resolution will be correlated for time-dependent measurements. The impact of ignoring these small correlations is a marginal underestimation of the uncertainties (assuming the correlation is positive), but given that the combination is still statistically dominated $\left(3.3^{\circ}\right.$ out of $3.6^{\circ}$ ) the effect is expected to be negligible.

\section{Statistical treatment}

The results are obtained using a frequentist treatment, with a likelihood function built from the product of the probability density functions, $f_{i}$, of experimental observables $\vec{A}_{i}$, 
defined as

$$
\mathcal{L}(\vec{\alpha})=\prod_{i} f_{i}\left(\vec{A}_{i}^{\mathrm{obs}} \mid \vec{\alpha}\right)
$$

Here, $\overrightarrow{A_{i}^{\text {obs }}}$ denotes the measured observables from analysis $i$, and $\vec{\alpha}$ is the set of underlying physics parameters on which they depend. The observables of each input are assumed to follow a multi-dimensional Gaussian distribution

$$
f_{i}\left(\vec{A}_{i}^{\mathrm{obs}} \mid \vec{\alpha}\right) \propto \exp \left(-\frac{1}{2}\left(\vec{A}_{i}(\vec{\alpha})-\vec{A}_{i}^{\mathrm{obs}}\right)^{T} V_{i}^{-1}\left(\vec{A}_{i}(\vec{\alpha})-\vec{A}_{i}^{\mathrm{obs}}\right)\right),
$$

where $V_{i}$ is the experimental covariance matrix, including both statistical and systematic uncertainties and their correlations.

A $\chi^{2}$-function is defined as $\chi^{2}(\vec{\alpha})=-2 \ln \mathcal{L}(\vec{\alpha})$, with the best-fit point given by the global minimum of the $\chi^{2}$ function, $\chi^{2}\left(\vec{\alpha}_{\text {min }}\right)$. The confidence level (CL) for a parameter at a given value, denoted $\alpha_{0}$, is determined in the following way. First, for every fixed $\alpha_{0}$, a new minimum of $\alpha^{\prime}$ is found, $\chi^{2}\left(\vec{\alpha}_{\min }^{\prime}\right)$, and the deviation from the global minimum, $\Delta \chi^{2}=\chi^{2}\left(\vec{\alpha}_{\min }^{\prime}\right)-\chi^{2}\left(\vec{\alpha}_{\min }\right)$, is computed. Second, an ensemble of pseudoexperiments, $\vec{A}_{j}^{\mathrm{MC}}$, is generated according to the probability distribution of eq. (3.2), with parameters $\vec{\alpha}=\vec{\alpha}_{\text {min }}^{\prime}$. Finally, for each pseudoexperiment the $\chi^{2}$-function is minimised once with the parameter of interest free to vary and once with it at a fixed value $\alpha_{0}$, to obtain the difference, $\left(\Delta \chi^{2}\right)^{\mathrm{MC}}$, from $\vec{A}_{j}^{\mathrm{MC}}$, in the same way as $\Delta \chi^{2}$ was computed from $\vec{A}_{i}^{\text {obs }}$. The $p$-value, or $1-\mathrm{CL}$, is then defined as the fraction of pseudoexperiments with $\left(\Delta \chi^{2}\right)^{\mathrm{MC}}>$ $\Delta \chi^{2}$. This method is often referred to as the $\hat{\mu}$ or Plugin method; see ref. [57] for details. Its coverage is not guaranteed [57] for the full parameter space, but can be evaluated at various points across the phase space. The coverage of the intervals quoted in this combination has been computed at several points across the phase space, including at the global minimum, by generating large samples of pseudoexperiments and computing the fraction which contains the generated value within a given confidence level. The coverage of the quoted $68.3 \%$ interval for $\gamma$ is $(67.3 \pm 1.5) \%$, for $x$ is $(68.2 \pm 1.5) \%$, for $y$ is $(67.6 \pm 1.5) \%$, for $|q / p|$ is $(66.6 \pm 1.5) \%$, and for $\phi$ is $(67.7 \pm 1.5) \%$. Similar coverage is seen for the $95.4 \%$ intervals and no correction to the quoted intervals is applied.

\section{Results}

The combination uses a total of 151 input observables to determine 52 free parameters, and the goodness of fit is found to be $84 \%$, evaluated using the best-fit $\chi^{2}$ and cross-checked with pseudoexperiments. The resulting confidence intervals for each parameter of interest, except for externally constrained nuisance parameters, are provided in table 3 . The correlation matrix of the parameters in table 3 is given in appendix $\mathrm{A}$, tables 7,8 and 9 . The $p$ value (or $1-\mathrm{CL}$ ) distribution as a function of $\gamma$ is shown in figure 2 for the total combination and for subsets in which the input observables are split by the species of the initial $B$ meson. The corresponding confidence intervals are provided in table 4. Significant differences between initial state $B$ mesons could be an indication of new physics entering at tree-level, as the decay topologies for charged and neutral initial states are different. Figure 2 shows a 


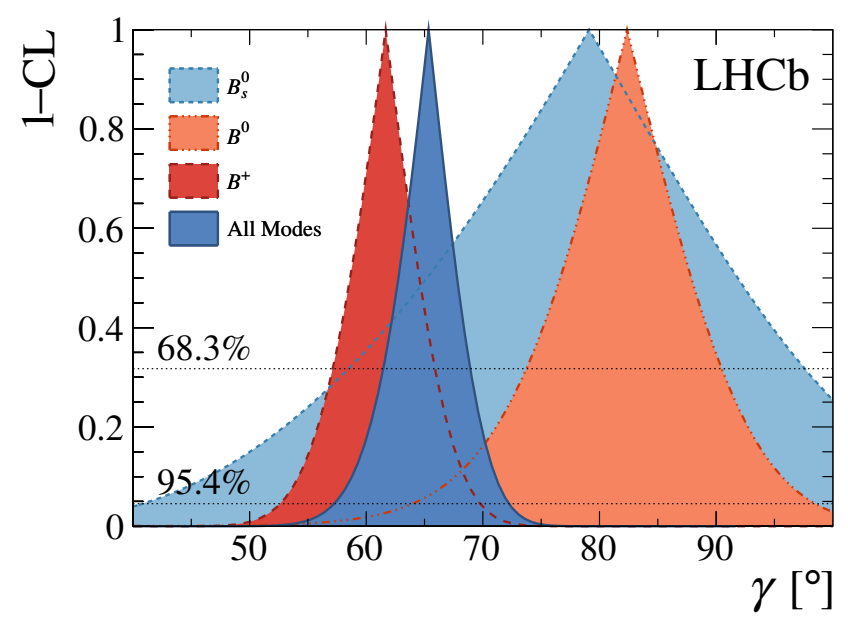

Figure 2. One dimensional 1 - CL profiles for $\gamma$ from the combination using inputs from $B_{s}^{0}$ (light blue), $B^{0}$ (orange), $B^{+}$mesons (red) and all species together (dark blue).
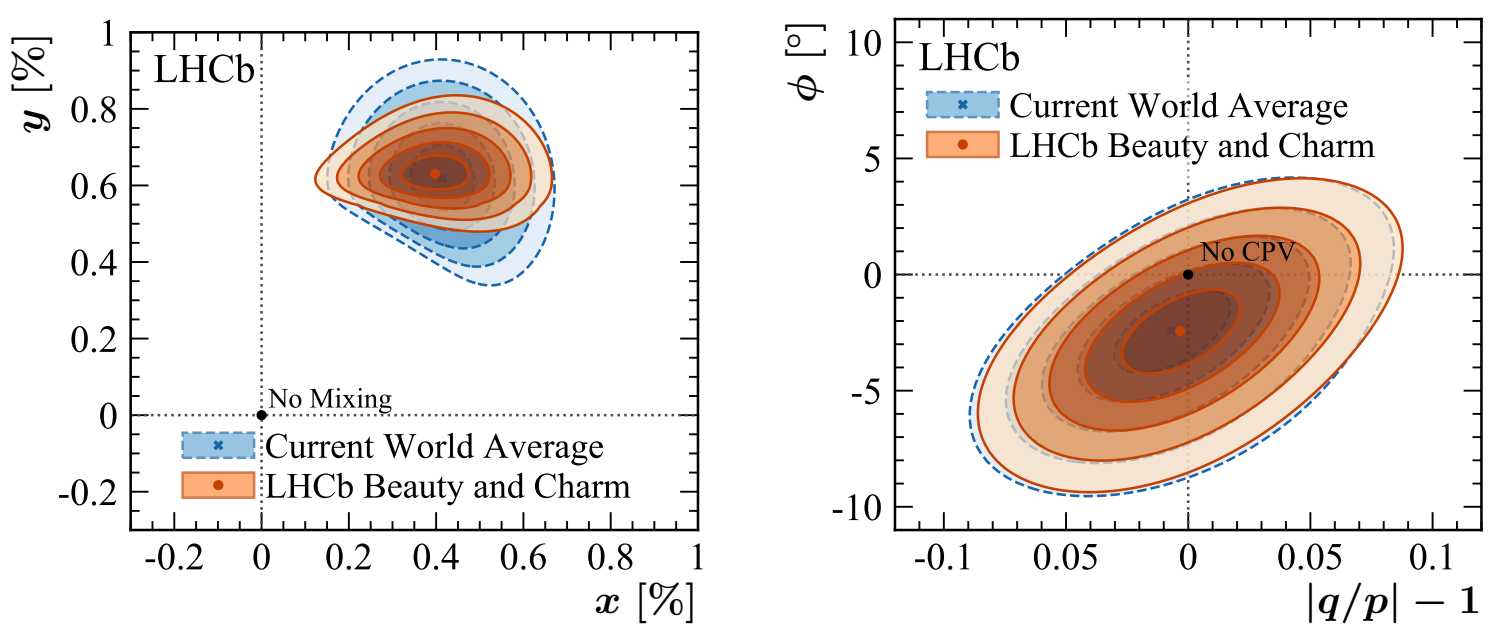

Figure 3. Two-dimensional profile likelihood contours for (left) the charm mixing parameters $x$ and $y$, and (right) the $\phi$ and $|q / p|$ parameters. The blue contours show the current charm world average from ref. [11]; the brown contours show the result of this combination. Contours are drawn out from $1(68.3 \%)$ to 5 standard deviations.

moderate tension, 2.2 standard deviations $(\sigma)$, between the charged and neutral $B$ states. The uncertainties in the $B^{0}$ and $B_{s}^{0}$ modes are considerably larger than in the dominant $B^{+}$ modes. The sensitivity of the $B^{0}$ and $B_{s}^{0}$ modes is expected to improve by approximately a factor of 2 with the analysis of $B^{0} \rightarrow D K^{+} \pi^{-}$with $D \rightarrow K_{\mathrm{S}}^{0} h^{+} h^{-}$and $B_{s}^{0} \rightarrow D_{s}^{\mp} K^{ \pm}$ decays using the full $\mathrm{LHCb}$ data sample. Table 5 presents the confidence intervals for $\gamma$ as determined from inputs of time-dependent methods and time-integrated methods only. Two-dimensional profile likelihood contours in the $(x, y)$ and $(|q / p|, \phi)$ planes are shown in figure 3. The significant improvement, of a factor of two, in the precision to $y$ demonstrates the advantage of this combination over the current world average in the charm system. 


\begin{tabular}{|c|c|c|c|c|c|}
\hline \multirow{2}{*}{ Quantity } & \multirow{2}{*}{ Value } & \multicolumn{2}{|r|}{$68.3 \% \mathrm{CL}$} & \multicolumn{2}{|r|}{$95.4 \% \mathrm{CL}$} \\
\hline & & Uncertainty & Interval & Uncertainty & Interval \\
\hline$\gamma\left[^{\circ}\right]$ & 65.4 & $\begin{array}{l}+3.8 \\
-4.2\end{array}$ & {$[61.2,69.2]$} & $\begin{array}{l}+7.5 \\
-8.7\end{array}$ & {$[56.7,72.9]$} \\
\hline$r_{B^{ \pm}}^{D K^{ \pm}}$ & 0.0984 & $\begin{array}{l}+0.0027 \\
-0.0026\end{array}$ & {$[0.0958,0.1011]$} & $\begin{array}{l}+0.0056 \\
-0.0052\end{array}$ & {$[0.0932,0.1040]$} \\
\hline$\delta_{B^{ \pm}}^{D K^{ \pm}}\left[{ }^{\circ}\right]$ & 127.6 & $\begin{array}{l}+4.0 \\
-4.2\end{array}$ & {$[123.4,131.6]$} & $\begin{array}{l}+7.8 \\
-9.2\end{array}$ & {$[118.4,135.4]$} \\
\hline$r_{B^{ \pm}}^{D \pi^{ \pm}}$ & 0.00480 & $\begin{array}{l}+0.00070 \\
-0.00056\end{array}$ & {$[0.00424,0.00550]$} & ${ }_{-0.0011}^{+0.0017}$ & {$[0.0037,0.0065]$} \\
\hline$\delta_{B^{ \pm}}^{D \pi^{ \pm}}\left[{ }^{\circ}\right]$ & 288 & $\begin{array}{l}+14 \\
-15\end{array}$ & {$[273,302]$} & $\begin{array}{l}+26 \\
-31\end{array}$ & {$[257,314]$} \\
\hline$r_{B^{ \pm}}^{D^{*} K^{ \pm}}$ & 0.099 & $\begin{array}{l}+0.016 \\
-0.019\end{array}$ & {$[0.080,0.115]$} & $\begin{array}{l}+0.030 \\
-0.038\end{array}$ & {$[0.061,0.129]$} \\
\hline$\delta_{B^{ \pm}}^{D^{*} K^{ \pm}}\left[{ }^{\circ}\right]$ & 310 & $\begin{array}{l}+12 \\
-23\end{array}$ & {$[287,322]$} & $\begin{array}{l}+20 \\
-71\end{array}$ & {$[239,330]$} \\
\hline$r_{B^{ \pm}}^{D^{*} \pi^{ \pm}}$ & 0.0095 & $\begin{array}{l}+0.0085 \\
-0.0061\end{array}$ & {$[0.0034,0.0180]$} & $\begin{array}{l}+0.017 \\
-0.0089\end{array}$ & {$[0.0006,0.026]$} \\
\hline$\delta_{B^{ \pm}}^{D^{*} \pi^{ \pm}}\left[{ }^{\circ}\right]$ & 139 & $\stackrel{+}{-86}^{22}$ & {$[53,161]$} & $\begin{array}{l}+32 \\
-129\end{array}$ & {$[10,171]$} \\
\hline$r_{B^{ \pm}}^{D K^{* \pm}}$ & 0.106 & $\begin{array}{l}+0.017 \\
-0.019\end{array}$ & {$[0.087,0.123]$} & $\begin{array}{l}+0.031 \\
-0.040\end{array}$ & {$[0.066,0.137]$} \\
\hline$\delta_{B^{ \pm}}^{D K^{* \pm}}\left[{ }^{\circ}\right]$ & 35 & $\begin{array}{l}+20 \\
-15\end{array}$ & {$[20,55]$} & $\begin{array}{l}+57 \\
-28\end{array}$ & {$[7,92]$} \\
\hline$r_{B^{0}}^{D K^{* 0}}$ & 0.250 & $\begin{array}{l}+0.023 \\
-0.024\end{array}$ & {$[0.226,0.273]$} & $\begin{array}{l}+0.044 \\
-0.052\end{array}$ & {$[0.198,0.294]$} \\
\hline$\delta_{B^{0}}^{D K^{* 0}}\left[{ }^{\circ}\right]$ & 197 & $\begin{array}{l}+10 \\
-9.3\end{array}$ & {$[187.7,207]$} & $\begin{array}{l}+24 \\
-18\end{array}$ & {$[179,221]$} \\
\hline$r_{B_{s}^{0}}^{D_{s}^{\mp} K^{ \pm}}$ & 0.310 & $\begin{array}{l}+0.098 \\
{ }_{-0.092}\end{array}$ & {$[0.218,0.408]$} & $\begin{array}{l}+0.20 \\
-0.21\end{array}$ & {$[0.10,0.51]$} \\
\hline$\delta_{B_{s}^{0}}^{D_{s}^{+}} K^{ \pm}\left[{ }^{\circ}\right]$ & 356 & $\begin{array}{l}+19 \\
-18\end{array}$ & {$[338,375]$} & $\begin{array}{l}+39 \\
+39\end{array}$ & {$[317,395]$} \\
\hline$r_{B_{s}^{0}}^{D^{\mp}} K^{ \pm} \pi^{+} \pi^{-}$ & 0.460 & $\begin{array}{l}+0.081 \\
-0.084\end{array}$ & {$[0.376,0.541]$} & $\begin{array}{l}+0.16 \\
-0.17\end{array}$ & {$[0.29,0.62]$} \\
\hline$\delta_{B_{s}^{0}}^{D_{s}^{s}} K^{ \pm} \pi^{+} \pi^{-}\left[{ }^{\circ}\right]$ & 345 & $\stackrel{+}{-12}^{+13}$ & {$[333,358]$} & $\begin{array}{l}+26 \\
-25\end{array}$ & {$[320,371]$} \\
\hline$r_{B^{0}}^{D^{\mp} \pi^{ \pm}}$ & 0.030 & $\begin{array}{l}+0.014 \\
-0.012\end{array}$ & {$[0.018,0.044]$} & $\begin{array}{l}+0.036 \\
-0.028\end{array}$ & {$[0.002,0.066]$} \\
\hline$\delta_{B^{0}}^{D^{\mp} \pi^{ \pm}}\left[{ }^{\circ}\right]$ & 30 & $\stackrel{+}{-37}^{+26}$ & {$[-7,56]$} & $\stackrel{+}{-81}^{+45}$ & {$[-51,75]$} \\
\hline$r_{B^{ \pm}}^{D K^{ \pm} \pi^{+} \pi^{-}}$ & 0.079 & $\begin{array}{l}+0.028 \\
-0.034\end{array}$ & {$[0.045,0.107]$} & $\begin{array}{l}+0.050 \\
-0.079\end{array}$ & {$[0.000,0.129]^{*}$} \\
\hline$r_{B^{ \pm}}^{D \pi^{ \pm}} \pi^{+} \pi^{-}$ & 0.067 & $\begin{array}{l}+0.025 \\
-0.029\end{array}$ & {$[0.038,0.092]$} & $\begin{array}{l}+0.040 \\
-0.067\end{array}$ & {$[0.000,0.107]^{*}$} \\
\hline$x[\%]$ & 0.400 & $\begin{array}{l}+0.052 \\
-0.053\end{array}$ & {$[0.347,0.452]$} & $\begin{array}{l}+0.10 \\
-0.11\end{array}$ & {$[0.29,0.50]$} \\
\hline$y[\%]$ & 0.630 & $\begin{array}{l}+0.033 \\
-0.030\end{array}$ & {$[0.600,0.663]$} & $\begin{array}{l}+0.069 \\
-0.058\end{array}$ & {$[0.572,0.699]$} \\
\hline$r_{D}^{K \pi}$ & 0.05867 & $\begin{array}{l}+0.00015 \\
-0.00015\end{array}$ & {$[0.05852,0.05882]$} & $\begin{array}{l}+0.00031 \\
-0.00030\end{array}$ & {$[0.05837,0.05898]$} \\
\hline$\delta_{D}^{K \pi}\left[^{\circ}\right]$ & 190.0 & $\begin{array}{l}+4.2 \\
-4.1\end{array}$ & {$[185.9,194.2]$} & $\begin{array}{l}+8.6 \\
-8.3\end{array}$ & {$[181.7,198.6]$} \\
\hline$r_{D}^{K_{\mathrm{S}}^{0} K \pi}$ & 0.6150 & $\begin{array}{l}+0.0056 \\
{ }_{-0} 0.0054\end{array}$ & {$[0.6096,0.6206]$} & ${ }_{-0.011}^{+0.011}$ & {$[0.604,0.626]$} \\
\hline$\delta_{D}^{K_{\mathrm{S}}^{0} K \pi}\left[{ }^{\circ}\right]$ & 17 & ${ }_{-14}^{+13}$ & {$[3,30]$} & $\begin{array}{l}+26 \\
-30\end{array}$ & {$[-13,43]$} \\
\hline$\kappa_{D}^{K_{\mathrm{S}}^{0} K \pi}$ & 0.82 & \pm 0.10 & {$[0.72,0.92]$} & \pm 0.20 & {$[0.62,1.02]$} \\
\hline$r_{D}^{K 3 \pi}$ & 0.05560 & $\begin{array}{l}+0.00059 \\
-0.00058\end{array}$ & {$[0.05502,0.05619]$} & ${ }_{-0.0012}^{+0.0012}$ & {$[0.0544,0.0568]$} \\
\hline$\delta_{D}^{K 3 \pi}\left[{ }^{\circ}\right]$ & 154 & $\begin{array}{l}+15 \\
-16\end{array}$ & {$[138,169]$} & $\begin{array}{l}+29 \\
-33\end{array}$ & {$[121,183]$} \\
\hline$\kappa_{D}^{K 3 \pi}$ & 0.480 & \pm 0.064 & {$[0.416,0.544]$} & \pm 0.13 & {$[0.35,0.60]$} \\
\hline$|q / p|$ & 0.997 & \pm 0.016 & {$[0.981,1.013]$} & \pm 0.033 & {$[0.964,1.030]$} \\
\hline$\phi\left[^{\circ}\right]$ & -2.4 & \pm 1.2 & {$[-3.6,-1.2]$} & \pm 2.5 & {$[-4.9,0.1]$} \\
\hline$\Delta a_{C P}^{\mathrm{dir}}$ & -0.00152 & \pm 0.00029 & {$[-0.00181,-0.00123]$} & \pm 0.00058 & {$[-0.00210,-0.00094]$} \\
\hline
\end{tabular}

Table 3. Confidence intervals and central values for each of the parameters of interest. Entries marked with an asterisk show where the scan has hit a physical boundary at the lower limit. 


\begin{tabular}{lccccc}
\hline \multirow{2}{*}{ Species } & \multirow{2}{*}{ Value ${ }^{\circ}{ }^{\circ}$} & \multicolumn{2}{c}{$68.3 \% \mathrm{CL}$} & \multicolumn{2}{c}{$95.4 \% \mathrm{CL}$} \\
& & Uncertainty & Interval & Uncertainty & Interval \\
\hline$B^{+}$ & 61.7 & ${ }_{-4.8}^{+4.4}$ & {$[56.9,66.1]$} & ${ }_{-9.5}^{+8.6}$ & {$[52.2,70.3]$} \\
$B^{0}$ & 82.0 & ${ }_{-8.8}^{+8.1}$ & {$[73.2,90.1]$} & ${ }_{-18}^{+17}$ & {$[64,99]$} \\
$B_{s}^{0}$ & 79 & ${ }_{-24}^{+21}$ & {$[55,100]$} & ${ }_{-47}^{+51}$ & {$[32,130]$} \\
\hline
\end{tabular}

Table 4. Confidence intervals and best-fit values for $\gamma$ when splitting the combination inputs by initial $B$ meson species.

\begin{tabular}{lccccc}
\hline \multirow{2}{*}{ Method } & \multirow{2}{*}{ Value $^{\circ}{ }^{\circ}$} & \multicolumn{2}{c}{$68.3 \%$ CL } & \multicolumn{2}{c}{$95.4 \%$ CL } \\
& & Uncertainty & Interval & Uncertainty & Interval \\
\hline Time-dependent & 79 & ${ }_{-24}^{+21}$ & {$[55,100]$} & ${ }_{-47}^{+51}$ & {$[32,130]$} \\
Time-integrated & 64.9 & ${ }_{-4.5}^{+3.9}$ & {$[60.4,68.8]$} & ${ }_{-9.6}^{+7.8}$ & {$[55.3,72.7]$} \\
\hline
\end{tabular}

Table 5. Confidence intervals and best-fit values for $\gamma$ when splitting the combination inputs by time-dependent and time-integrated methods.

Breakdowns of the contributing components in the combination are shown in figures 4 and 5. These highlight the complementary nature of the input measurements to constrain both $\gamma$ and the charm mixing parameters. In figure 5 (top left) the dark orange band shows external constraints from CLEO-c [58] and BES-III [59]. These are required to constrain $\delta_{D}^{K \pi}$ when obtaining the "All Charm Modes" contours, but are not used in the full combination. In the top right and bottom plots the orange bands show the constraints from $D^{0} \rightarrow h^{+} h^{-}$modes, but these cannot provide bands in $(x, y)$ or $(|q / p|, \phi)$ without other constraints [60]. Consequently, when these orange bands are produced in the top right plot $\left(|q / p|, \phi, r_{D}^{K \pi}, \delta_{D}^{K \pi}\right)$ are fixed to their best fit values from table 3 , while in the bottom plot $\left(x, y, r_{D}^{K \pi}, \delta_{D}^{K \pi}\right)$ are fixed to their best fit values. In the bottom figure the red contour is mostly hidden behind the blue; this is because no significant additional sensitivity to $C P$ violation in the charm system is provided by the inclusion of the beauty observables in the simultaneous fit.

The value of $\gamma=\left(65.4_{-4.2}^{+3.8}\right)^{\circ}$ determined from this combination is compatible with, but lower than that of the previous LHCb combination $\gamma=\left(74_{-5.8}^{+5.0}\right)^{\circ}[17]$. This change is driven by improved treatments of background sources in the major inputs described in refs. [19, 20]. An assessment of the compatibility between this and the previous combination, which considers the full parameter space and the correlation between the current set of inputs and the previous set of inputs, finds they are compatible at the level of $2.1 \sigma$. The new result is in excellent agreement with the global CKM fit results [12, 13].

The charm mixing parameters, $x$ and $y$, are determined simultaneously with $\gamma$ in this combination for the first time. The precision on $x$ is driven by the recent measurement described in ref. [44]. The result $y=\left(0.630_{-0.030}^{+0.033}\right) \%$ is more precise than the world average, $y=\left(0.603_{-0.056}^{+0.057}\right) \%$ [11], by approximately a factor of two, driven entirely by the improved measurement of $\delta_{D}^{K \pi}$ from the beauty system and the simultaneous averaging methodology 

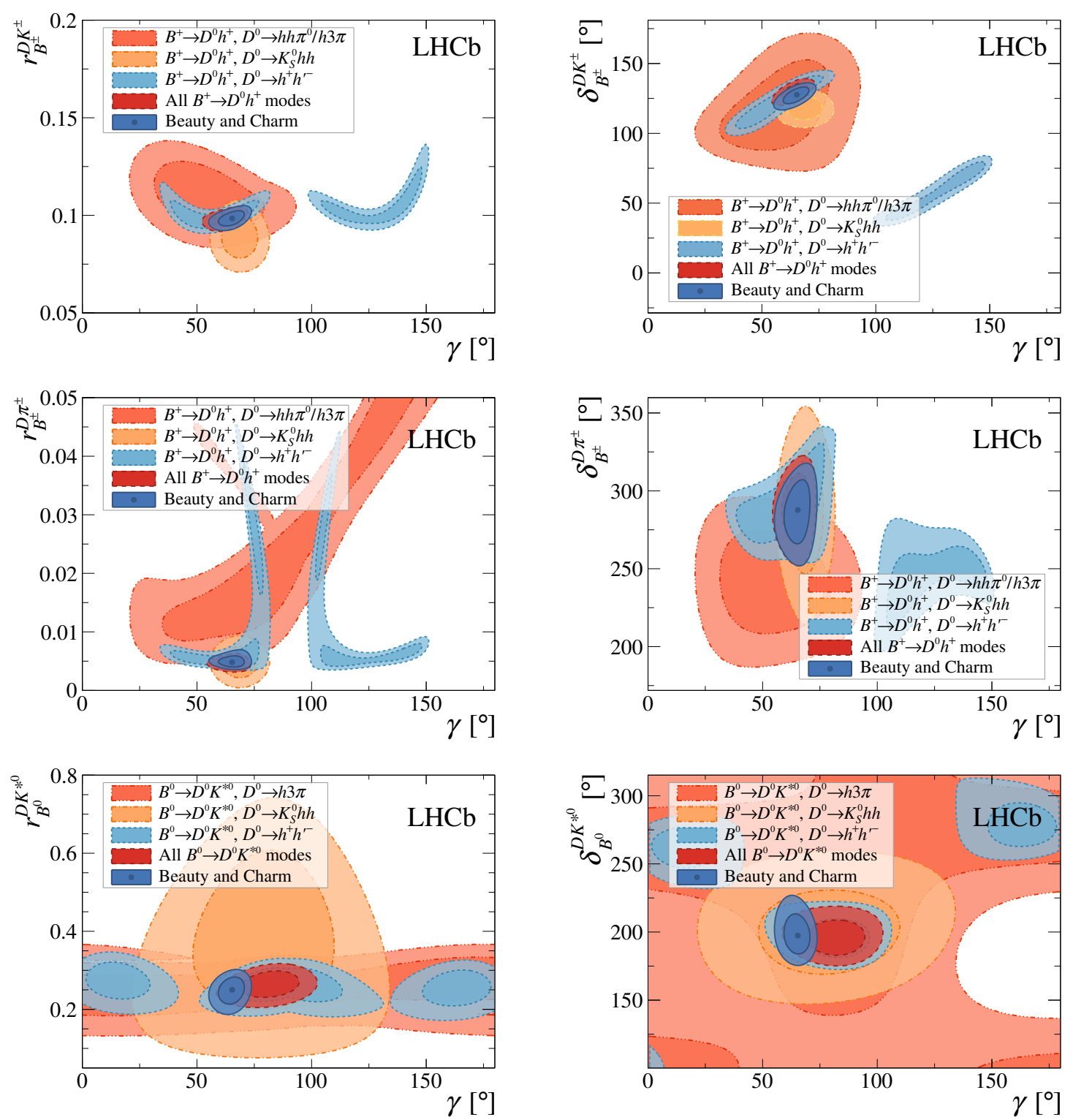

Figure 4. Profile likelihood contours for the beauty decay parameters versus $\gamma$, showing the breakdown of sensitivity amongst different sub-combinations of modes. The contours indicate the $68.3 \%$ and $95.4 \%$ confidence region.

employed in this article. The correlation between $\delta_{D}^{K \pi}$ and $\delta_{B^{ \pm}}^{D K^{ \pm}}$is $-57 \%$, highlighting $B^{ \pm} \rightarrow D K^{ \pm}$decays as the source of this improvement.

The beauty part of the combination is cross-checked with an independent framework using a Bayesian statistical treatment. A flat prior is used for $\gamma$ and the relevant hadronic parameters and results in a value of $\gamma=\left(65.6_{-3.8}^{+3.7}\right)^{\circ}$, in agreement with the default frequentist results. Good agreement between the frequentist and Bayesian interpretations is also seen for the other hadronic parameters. A second cross-check using an independent fitting framework with frequentist interpretation gives consistent results to better than 

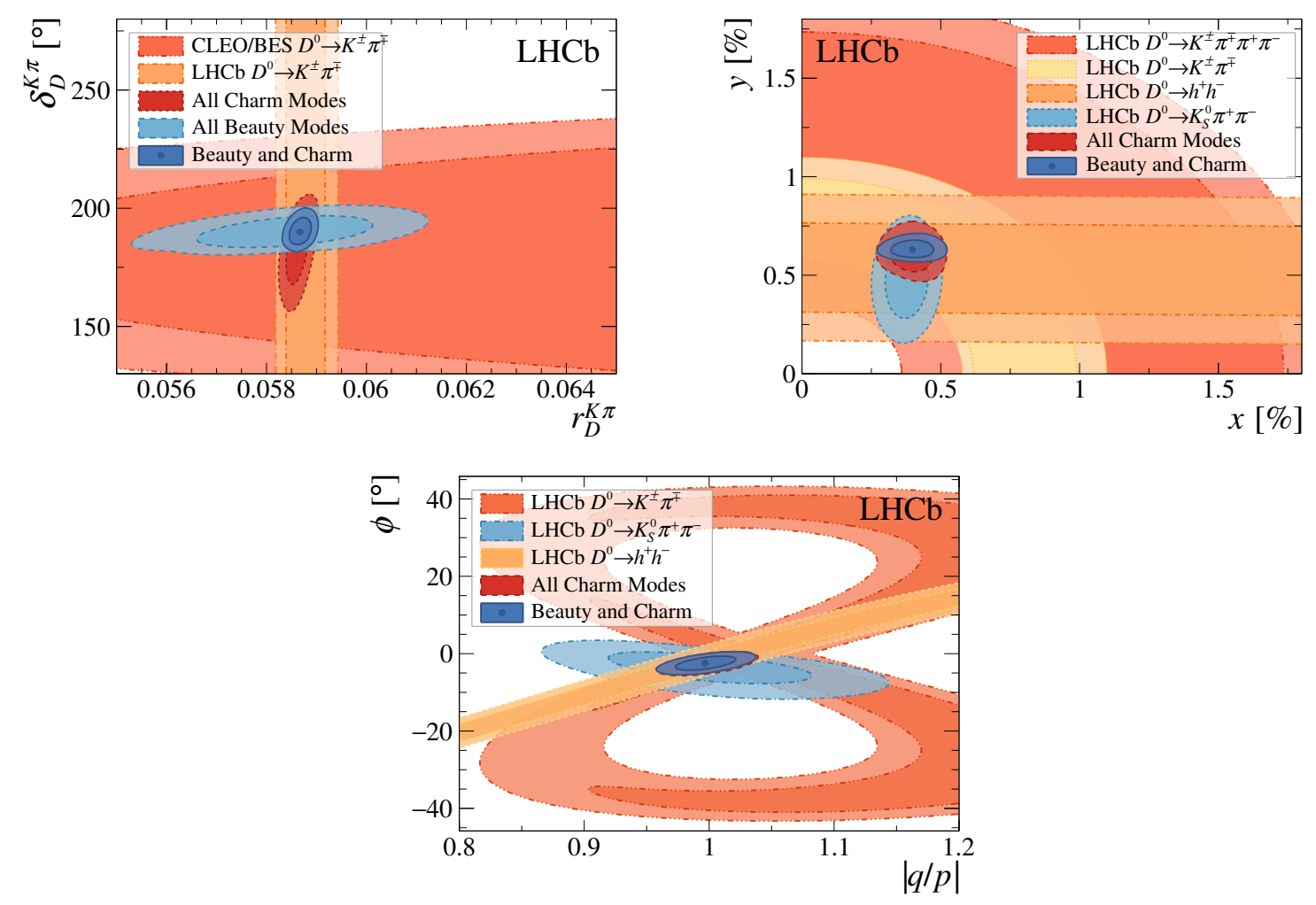

Figure 5. Profile likelihood contours for the charm decay parameters, showing the breakdown of sensitivity amongst different sub-combinations of modes. The contours indicate the $68.3 \%$ and $95.4 \%$ confidence region.

$1 \%$ precision. Finally, the charm sector of the combination was validated by accurately reproducing the HFLAV results [11].

The relative impact of systematic uncertainties on the input observables is studied, and found to contribute approximately $1.2^{\circ}$ to the result for $\gamma$, demonstrating that the uncertainty of this combination is still dominated by the data sample size.

In previous combinations, the experimental input from $B^{0} \rightarrow D^{\mp} \pi^{ \pm}$decays was included with an external theoretical prediction of $r_{B^{0}}^{D^{\mp} \pi^{ \pm}}=0.0182 \pm 0.0038$ [28]. This prediction assumes SU(3) symmetry, and was the only input from theory. This external input is no longer used, and the combination gives an experimental determination of $r_{B^{0}}^{D^{\mp} \pi^{ \pm}}=0.030_{-0.012}^{+0.014}$. This is in agreement with the theory-based prediction and provides confidence that the assumption of $\mathrm{SU}(3)$ symmetry is valid within the current precision. This change has a negligible impact on the determination of other parameters.

\section{Conclusion}

A simultaneous combination of LHCb measurements sensitive to the CKM angle $\gamma$ and charm mixing parameters, along with auxiliary information from other experiments, is performed for the first time. This includes seven new and updated inputs from $B$-meson 
decays and eight inputs from $D$-meson decays. The result,

$$
\gamma=\left(65.4_{-4.2}^{+3.8}\right)^{\circ}
$$

provides the most precise measurement from a single experiment. The charm mixing parameters are found to be

$$
\begin{aligned}
& x=\left(0.400_{-0.053}^{+0.052}\right) \%, \\
& y=\left(0.630_{-0.030}^{+0.033}\right) \%,
\end{aligned}
$$

which are the most precise determinations to date. In particular, the uncertainty on $y$ is reduced by a factor of two by using the new procedure described in this paper.

\section{Acknowledgments}

We express our gratitude to our colleagues in the CERN accelerator departments for the excellent performance of the LHC. We thank the technical and administrative staff at the LHCb institutes. We acknowledge support from CERN and from the national agencies: CAPES, CNPq, FAPERJ and FINEP (Brazil); MOST and NSFC (China); CNRS/IN2P3 (France); BMBF, DFG and MPG (Germany); INFN (Italy); NWO (Netherlands); MNiSW and NCN (Poland); MEN/IFA (Romania); MSHE (Russia); MICINN (Spain); SNSF and SER (Switzerland); NASU (Ukraine); STFC (United Kingdom); DOE NP and NSF (U.S.A.). We acknowledge the computing resources that are provided by CERN, IN2P3 (France), KIT and DESY (Germany), INFN (Italy), SURF (Netherlands), PIC (Spain), GridPP (United Kingdom), RRCKI and Yandex LLC (Russia), CSCS (Switzerland), IFINHH (Romania), CBPF (Brazil), PL-GRID (Poland) and NERSC (U.S.A.). We are indebted to the communities behind the multiple open-source software packages on which we depend. Individual groups or members have received support from ARC and ARDC (Australia); AvH Foundation (Germany); EPLANET, Marie Skłodowska-Curie Actions and ERC (European Union); A*MIDEX, ANR, Labex P2IO and OCEVU, and Région Auvergne-RhôneAlpes (France); Key Research Program of Frontier Sciences of CAS, CAS PIFI, CAS CCEPP, Fundamental Research Funds for the Central Universities, and Sci. \& Tech. Program of Guangzhou (China); RFBR, RSF and Yandex LLC (Russia); GVA, XuntaGal and GENCAT (Spain); the Leverhulme Trust, the Royal Society and UKRI (United Kingdom).

\section{A Correlation matrix}

The global fit correlation matrix between each of the parameters presented in table 3 is provided in tables 7, 8 and 9. A subset of this matrix, including only the parameters of greatest interest, is given in table 6 . The correlation coefficients for $\beta$ and $\phi_{s}$ are not included as they are almost all smaller than 0.001, an exception is $\rho\left(\gamma, \phi_{s}\right)=-0.009$. 


\begin{tabular}{lrrrrrrrr}
\hline & $\gamma$ & $A_{D}$ & $r_{D}^{K \pi}$ & $\delta_{D}^{K \pi}$ & $x$ & $y$ & $|q / p|$ & $\phi$ \\
\hline$\gamma$ & 1.000 & - & -0.003 & 0.003 & -0.002 & 0.003 & - & - \\
$A_{D}$ & & 1.000 & -0.016 & -0.083 & -0.003 & -0.083 & -0.316 & 0.295 \\
$r_{D}^{K \pi}$ & & & 1.000 & 0.295 & 0.282 & -0.095 & -0.070 & 0.015 \\
$\delta_{D}^{K \pi}$ & & & & 1.000 & 0.029 & 0.891 & -0.020 & 0.055 \\
$x$ & & & & & 1.000 & 0.013 & -0.129 & 0.083 \\
$y$ & & & & & & 1.000 & -0.018 & 0.040 \\
$|q / p|$ & & & & & & & 1.000 & 0.554 \\
$\phi$ & & & & & & & & 1.000 \\
\hline
\end{tabular}

Table 6. Reduced correlation matrix for the parameters of greater interest. Values smaller than 0.001 are replaced with a - symbol.

\begin{tabular}{|c|c|c|c|c|c|c|c|c|c|c|c|c|c|c|}
\hline & $\gamma$ & $r_{B^{ \pm}}^{D K^{ \pm}}$ & $\delta_{B^{ \pm}}^{D K^{ \pm}}$ & $r_{B^{ \pm}}^{D \pi^{ \pm}}$ & $\delta_{B^{ \pm}}^{D \pi^{ \pm}}$ & $r_{B^{ \pm}}^{D^{*} K^{ \pm}}$ & $\delta_{B^{ \pm}}^{D^{*} K^{ \pm}}$ & $r_{B^{ \pm}}^{D^{*} \pi^{ \pm}}$ & $\delta_{B^{ \pm}}^{D^{*} \pi^{ \pm}}$ & $r_{B^{ \pm}}^{D K^{* \pm}}$ & $\delta_{B^{ \pm}}^{D K^{* \pm}}$ & $r_{B^{0}}^{D K^{* 0}}$ & $\delta_{B^{0}}^{D K^{* 0}}$ & $r_{B_{s}^{0}}^{D^{+} K^{ \pm}}$ \\
\hline$\gamma$ & 1.000 & 0.490 & 0.613 & -0.051 & 0.229 & 0.138 & 0.331 & 0.116 & 0.149 & -0.158 & -0.012 & 0.206 & -0.111 & -0.003 \\
\hline$r_{B^{ \pm}}^{D K^{ \pm}}$ & & 1.000 & 0.442 & -0.048 & 0.113 & 0.058 & 0.174 & 0.070 & 0.100 & -0.079 & 0.026 & 0.098 & -0.047 & -0.001 \\
\hline$\delta_{B^{ \pm}}^{D K^{ \pm}}$ & & & 1.000 & -0.055 & 0.236 & 0.062 & 0.231 & 0.102 & 0.156 & -0.094 & 0.054 & 0.120 & -0.052 & -0.002 \\
\hline$r_{B^{ \pm}}^{D \pi^{ \pm}}$ & & & & 1.000 & 0.629 & -0.006 & -0.018 & -0.008 & -0.011 & 0.006 & -0.003 & -0.009 & 0.004 & - \\
\hline$\delta_{B^{ \pm}}^{D \pi^{ \pm}}$ & & & & & 1.000 & 0.025 & 0.085 & 0.034 & 0.052 & -0.035 & 0.011 & 0.046 & -0.022 & - \\
\hline$r_{B^{ \pm}}^{D^{*} K^{ \pm}}$ & & & & & & 1.000 & 0.764 & -0.211 & -0.213 & -0.022 & -0.006 & 0.029 & -0.016 & - \\
\hline$\delta_{B^{ \pm}}^{D^{*} K^{ \pm}}$ & & & & & & & 1.000 & -0.172 & -0.164 & -0.052 & 0.001 & 0.067 & -0.035 & - \\
\hline$r_{B^{ \pm}}^{D^{*} \pi^{ \pm}}$ & & & & & & & & 1.000 & 0.895 & -0.018 & 0.004 & 0.023 & -0.011 & - \\
\hline$\delta_{B^{ \pm}}^{D^{*} \pi^{ \pm}}$ & & & & & & & & & 1.000 & -0.023 & 0.010 & 0.029 & -0.013 & - \\
\hline$r_{B^{ \pm}}^{D K^{* \pm}}$ & & & & & & & & & & 1.000 & -0.323 & -0.033 & 0.017 & - \\
\hline$\delta_{B^{ \pm}}^{D K^{* \pm}}$ & & & & & & & & & & & 1.000 & -0.004 & 0.006 & - \\
\hline$r_{B^{0}}^{D K^{* 0}}$ & & & & & & & & & & & & 1.000 & -0.139 & - \\
\hline$\delta_{B^{0}}^{D K^{* 0}}$ & & & & & & & & & & & & & 1.000 & - \\
\hline$r_{B_{s}^{0}}^{D_{s}^{\mp} K^{ \pm}}$ & & & & & & & & & & & & & & 1.000 \\
\hline
\end{tabular}

Table 7. Correlation matrix of the fit result, part 1 of 3 . Values smaller than 0.001 are replaced with a - symbol.

\begin{tabular}{|c|c|c|c|c|c|c|c|c|c|c|c|c|c|}
\hline & $\begin{array}{l}\delta_{B_{s}^{0}}^{D_{s}^{\mp} K^{ \pm}} \\
\end{array}$ & $\begin{array}{l}r_{B_{s}^{0}}^{D_{s}^{\mp} K^{ \pm} \pi^{+} \pi^{-}} \\
\end{array}$ & $\delta_{B_{s}^{0}}^{D_{s}^{\mp} K^{ \pm} \pi^{+} \pi^{-}}$ & $r_{B^{0}}^{D^{\mp} \pi^{ \pm}}$ & $\delta_{B^{0}}^{D^{\mp} \pi^{ \pm}}$ & $r_{B^{ \pm}}^{D K^{ \pm} \pi^{+} \pi^{-}}$ & $r_{B^{ \pm}}^{D \pi^{ \pm} \pi^{+} \pi^{-}}$ & $r_{D}^{K \pi}$ & $\delta_{D}^{K \pi}$ & $x$ & $y$ & $|q / p|$ & $\phi$ \\
\hline$\gamma$ & -0.019 & -0.027 & -0.144 & -0.065 & -0.168 & 0.024 & -0.181 & -0.003 & 0.003 & -0.002 & 0.003 & - & - \\
\hline$r_{B^{ \pm}}^{D K^{ \pm}}$ & -0.009 & -0.013 & -0.071 & -0.032 & -0.082 & -0.006 & 0.038 & -0.079 & -0.236 & 0.011 & -0.206 & 0.003 & -0.010 \\
\hline$\delta_{B^{ \pm}}^{D K^{ \pm}}$ & -0.012 & -0.017 & -0.088 & -0.040 & -0.103 & -0.028 & 0.196 & -0.161 & -0.573 & 0.020 & -0.510 & 0.007 & -0.029 \\
\hline$r_{B^{ \pm}}^{D \pi^{ \pm}}$ & - & 0.001 & 0.007 & 0.003 & 0.009 & - & -0.003 & 0.084 & 0.022 & -0.005 & -0.004 & -0.005 & -0.004 \\
\hline$\delta_{B^{ \pm}}^{D \pi^{ \pm}}$ & -0.004 & -0.006 & -0.033 & -0.015 & -0.038 & -0.007 & 0.048 & 0.048 & -0.169 & - & -0.180 & -0.004 & -0.016 \\
\hline$r_{B^{ \pm}}^{D^{*} K^{ \pm}}$ & -0.003 & -0.004 & -0.020 & -0.009 & -0.023 & 0.006 & -0.046 & 0.013 & 0.040 & -0.002 & 0.035 & - & 0.002 \\
\hline$\delta_{B^{ \pm}}^{D^{*} K^{ \pm}}$ & -0.006 & -0.009 & -0.048 & -0.022 & -0.055 & 0.004 & -0.034 & -0.012 & -0.048 & - & -0.044 & - & -0.003 \\
\hline$r_{B^{ \pm}}^{D^{*} \pi^{ \pm}}$ & -0.002 & -0.003 & -0.017 & -0.008 & -0.019 & -0.001 & 0.008 & -0.024 & -0.053 & 0.002 & -0.045 & 0.001 & -0.002 \\
\hline$\delta_{B^{ \pm}}^{D^{*} \pi^{ \pm}}$ & -0.003 & -0.004 & -0.021 & -0.010 & -0.025 & -0.005 & 0.033 & -0.039 & -0.112 & 0.004 & -0.097 & 0.002 & -0.005 \\
\hline$r_{B^{ \pm}}^{D K^{* \pm}}$ & 0.003 & 0.004 & 0.023 & 0.010 & 0.026 & -0.004 & 0.032 & -0.003 & -0.006 & -0.002 & -0.005 & - & - \\
\hline$\delta_{B^{ \pm}}^{D K^{* \pm}}$ & - & - & 0.002 & - & 0.002 & -0.008 & 0.057 & -0.027 & -0.102 & 0.011 & -0.091 & - & -0.004 \\
\hline$r_{B^{0}}^{D K^{* 0}}$ & -0.004 & -0.006 & -0.030 & -0.013 & -0.034 & 0.006 & -0.043 & 0.002 & 0.011 & -0.002 & 0.010 & - & - \\
\hline$\delta_{B^{0}}^{D K^{* 0}}$ & 0.002 & 0.003 & 0.016 & 0.007 & 0.019 & -0.005 & 0.035 & -0.007 & -0.028 & 0.002 & -0.025 & - & -0.001 \\
\hline$r_{B_{s}^{0}}^{D_{s}^{\mp} K^{ \pm}}$ & -0.034 & - & - & - & - & - & - & - & - & - & - & - & - \\
\hline
\end{tabular}

Table 8. Correlation matrix of the fit result, part 2 of 3 . Values smaller than 0.001 are replaced with a - symbol. 


\begin{tabular}{|c|c|c|c|c|c|c|c|c|c|c|c|c|c|}
\hline & $\delta_{B_{s}^{0}}^{D^{\mp} K^{ \pm}}$ & $r_{B_{s}^{0}}^{D_{s}^{\mp} K^{ \pm} \pi^{+} \pi^{-}}$ & $\delta_{B_{s}^{0}}^{D_{s}^{\mp} K^{ \pm} \pi^{+} \pi^{-}}$ & $r_{B^{0}}^{D^{\mp} \pi^{ \pm}}$ & $\delta_{B^{0}}^{D^{\mp} \pi^{ \pm}}$ & $r_{B^{ \pm}}^{D K^{ \pm} \pi^{+} \pi^{-}}$ & $r_{B^{ \pm}}^{D \pi^{ \pm} \pi^{+} \pi^{-}}$ & $r_{D}^{K \pi}$ & $\delta_{D}^{K \pi}$ & $x$ & $y$ & $|q / p|$ & $\phi$ \\
\hline$\overline{\delta_{B_{s}^{0}}^{D_{s}^{+} K^{ \pm}}}$ & 1.000 & - & 0.003 & 0.001 & 0.003 & - & 0.003 & - & - & - & - & - & - \\
\hline$r_{B_{s}^{0}}^{D_{s}^{+} K^{ \pm} \pi^{+} \pi^{-}}$ & & 1.000 & 0.006 & 0.002 & 0.005 & - & 0.005 & - & - & - & - & - & - \\
\hline$\delta_{B_{s}^{0}}^{D_{s}^{+}} K^{ \pm} \pi^{+} \pi^{-}$ & & & 1.000 & 0.009 & 0.024 & -0.003 & 0.026 & - & - & - & - & - & - \\
\hline$r_{B^{0}}^{D^{5} \pi^{ \pm}}$ & & & & 1.000 & 0.485 & -0.002 & 0.012 & - & - & - & - & - & - \\
\hline$\delta_{B^{0}}^{D^{\mp} \pi^{ \pm}}$ & & & & & 1.000 & -0.004 & 0.030 & - & - & - & - & - & - \\
\hline$r_{B^{ \pm}}^{D K^{ \pm} \pi^{+} \pi^{-}}$ & & & & & & 1.000 & -0.044 & 0.020 & 0.074 & -0.003 & 0.066 & - & 0.004 \\
\hline$r_{B^{ \pm}}^{D \pi^{ \pm} \pi^{+} \pi^{-}}$ & & & & & & & 1.000 & -0.148 & -0.531 & 0.025 & -0.473 & 0.005 & -0.026 \\
\hline$r_{D}^{K \pi}$ & & & & & & & & 1.000 & 0.295 & 0.282 & -0.095 & -0.070 & 0.015 \\
\hline$\delta_{D}^{K \pi}$ & & & & & & & & & 1.000 & 0.029 & 0.892 & -0.020 & 0.055 \\
\hline$x$ & & & & & & & & & & 1.000 & 0.013 & -0.129 & 0.083 \\
\hline$y$ & & & & & & & & & & & 1.000 & -0.018 & 0.040 \\
\hline$|q / p|$ & & & & & & & & & & & & 1.000 & 0.554 \\
\hline$\phi$ & & & & & & & & & & & & & 1.000 \\
\hline
\end{tabular}

Table 9. Correlation matrix of the fit result, part 3 of 3 . Values smaller than 0.001 are replaced with a - symbol. 


\begin{tabular}{|c|c|c|c|}
\hline & Measurement & $\chi^{2}$ & No. of obs. \\
\hline \multirow{13}{*}{ 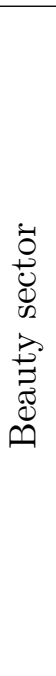 } & $B^{ \pm} \rightarrow D h^{ \pm}, D \rightarrow h^{ \pm} h^{\prime \mp}$ & 2.71 & 8 \\
\hline & $B^{ \pm} \rightarrow D h^{ \pm}, D \rightarrow h^{ \pm} \pi^{\mp} \pi^{+} \pi^{-}$ & 7.36 & 8 \\
\hline & $B^{ \pm} \rightarrow D h^{ \pm}, D \rightarrow h^{ \pm} h^{\prime \mp} \pi^{0}$ & 7.14 & 11 \\
\hline & $B^{ \pm} \rightarrow D h^{ \pm}, D \rightarrow K_{S}^{0} h^{+} h^{-}$ & 4.67 & 6 \\
\hline & $B^{ \pm} \rightarrow D h^{ \pm}, D \rightarrow K_{S}^{0} K^{ \pm} \pi^{\mp}$ & 7.57 & 7 \\
\hline & $B^{ \pm} \rightarrow D^{*} h^{ \pm}, D \rightarrow h^{ \pm} h^{\prime \mp}$ & 7.31 & 16 \\
\hline & $B^{ \pm} \rightarrow D K^{* \pm}, D \rightarrow h^{ \pm} h^{\prime \mp}\left(\pi^{+} \pi^{-}\right)$ & 3.71 & 12 \\
\hline & $B^{0} \rightarrow D K^{* 0}, D \rightarrow h^{ \pm} h^{\prime \mp}\left(\pi^{+} \pi^{-}\right)$ & 9.45 & 12 \\
\hline & $B^{0} \rightarrow D K^{* 0}, D \rightarrow K_{S}^{0} h^{+} h^{-}$ & 3.26 & 4 \\
\hline & $B^{ \pm} \rightarrow D h^{ \pm} \pi^{+} \pi^{-}, D \rightarrow h^{ \pm} h^{\prime \mp}$ & 1.34 & 11 \\
\hline & $B_{s}^{0} \rightarrow D_{s}^{\mp} K^{ \pm}$ & 5.71 & 5 \\
\hline & $B_{s}^{0} \rightarrow D_{s}^{\mp} K^{ \pm} \pi^{+} \pi^{-}$ & 2.88 & 5 \\
\hline & $B^{0} \rightarrow D^{\mp} \pi^{ \pm}$ & 0.00 & 2 \\
\hline \multirow{9}{*}{ 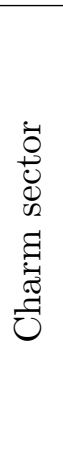 } & $D \rightarrow K_{S}^{0} \pi^{+} \pi^{-} 2011$ & 5.38 & 2 \\
\hline & $D \rightarrow K_{S}^{0} \pi^{+} \pi^{-}$Run 1 & 0.77 & 4 \\
\hline & $D \rightarrow K_{S}^{0} \pi^{+} \pi^{-}$Run 2 & 1.37 & 4 \\
\hline & $D \rightarrow K^{ \pm} \pi^{\mp}$ Run 1 & 1.29 & 6 \\
\hline & $D \rightarrow h^{+} h^{-} \Delta A_{C P}$ & 0.00 & 2 \\
\hline & $D \rightarrow K^{ \pm} \pi^{\mp} \pi^{+} \pi^{-}$ & 3.59 & 1 \\
\hline & $D \rightarrow h^{+} h^{-} y_{C P}$ & 0.40 & 1 \\
\hline & $D \rightarrow h^{+} h^{-} \Delta Y$ & 0.15 & 1 \\
\hline & $D \rightarrow K^{ \pm} \pi^{\mp} \operatorname{Run} 2$ & 2.23 & 6 \\
\hline \multirow{10}{*}{ 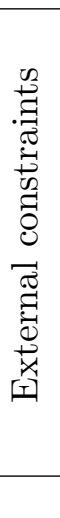 } & $D \rightarrow K^{ \pm} \pi^{\mp} \pi^{0}, D \rightarrow K^{ \pm} \pi^{\mp} \pi^{+} \pi^{-}$ & 0.79 & 6 \\
\hline & $D \rightarrow \pi^{+} \pi^{-} \pi^{+} \pi^{-}$ & 0.03 & 1 \\
\hline & $D \rightarrow h^{+} h^{-} \pi^{0}$ & 0.01 & 2 \\
\hline & $D \rightarrow K_{S}^{0} K^{ \pm} \pi^{\mp} \mathrm{WS}$ & 0.60 & 1 \\
\hline & $D \rightarrow K_{S}^{0} K^{ \pm} \pi^{\mp}$ & 3.79 & 3 \\
\hline & $B^{ \pm} \rightarrow D K^{* \pm}$ & 0.02 & 1 \\
\hline & $B^{0} \rightarrow D K^{* 0}$ & 0.01 & 1 \\
\hline & $\phi_{s}$ & 0.00 & 1 \\
\hline & $\beta$ & 0.00 & 1 \\
\hline & Total & 83.53 & 151 \\
\hline
\end{tabular}

Table 10. Contributions to the total $\chi^{2}$ and the number of observables of each input measurement.

\section{B Contribution of each input measurement to the global $\chi^{2}$}

The contribution of each input measurement to the global $\chi^{2}$ is shown in table 10 .

\section{Pull distribution of each input observable}

The pull of each input observable with respect to the global best fit point is shown in figures 6-8. The pull is defined as $\left(A_{\exp }-A_{\text {fit }}\right) / \sigma\left(A_{\exp }\right)$, where $A_{\exp }$ and $A_{\text {fit }}$ are the 

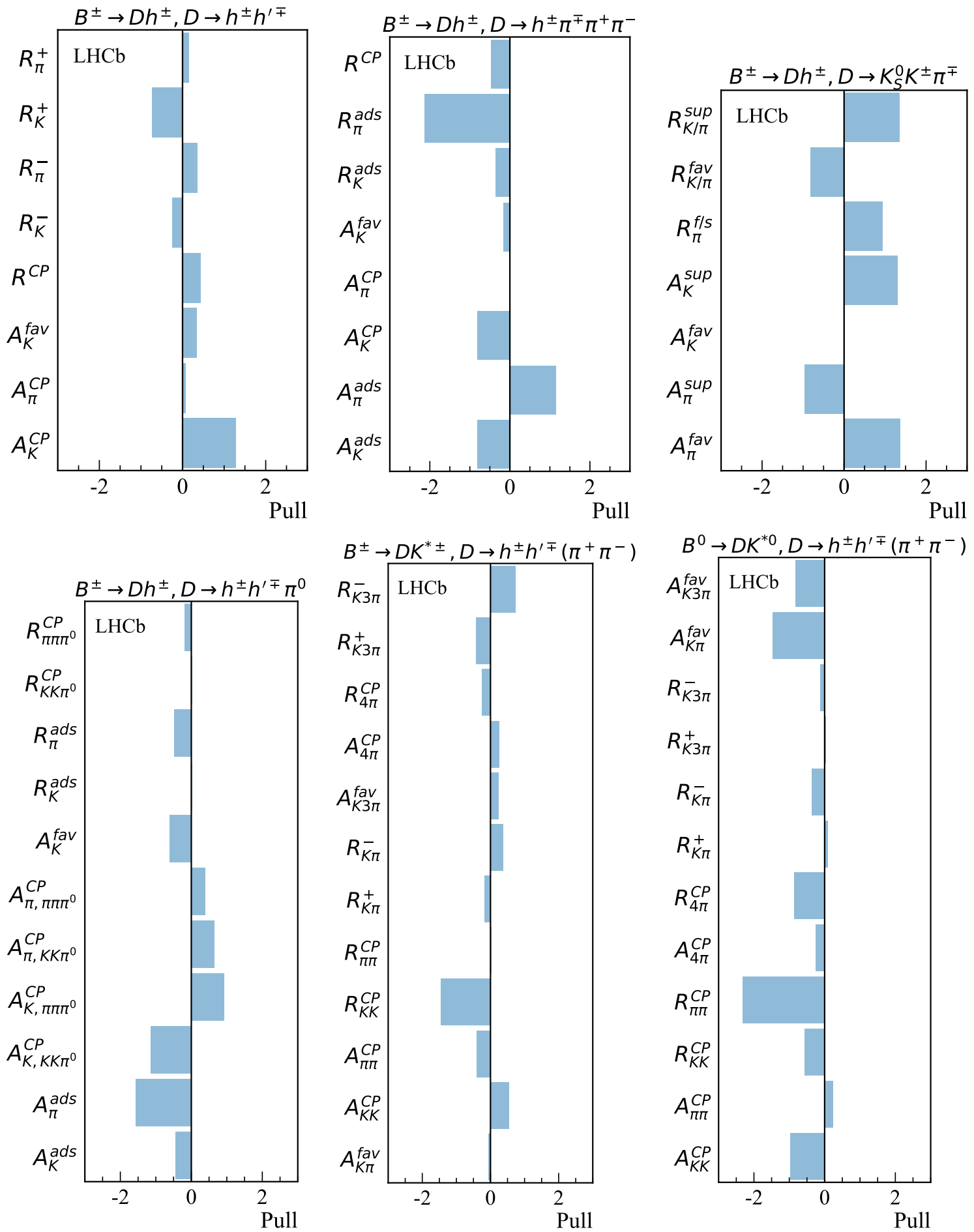

Figure 6. Pulls of the input observables, part 1 of 3.

experimental input value and value at the best-fit point, respectively, and $\sigma\left(A_{\exp }\right)$ is the experimental uncertainty. 

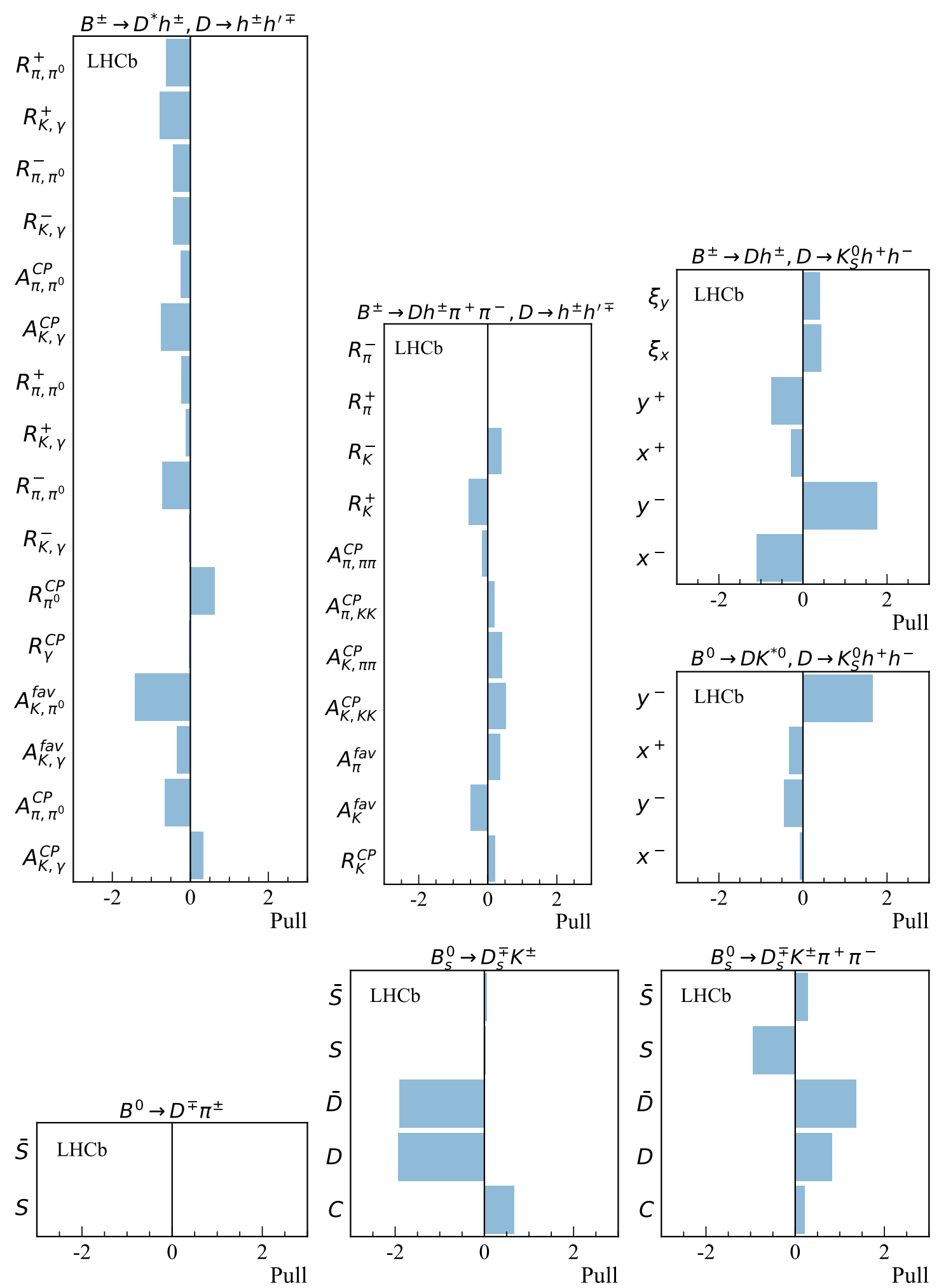

Figure 7. Pulls of the input observables, part 2 of 3. 

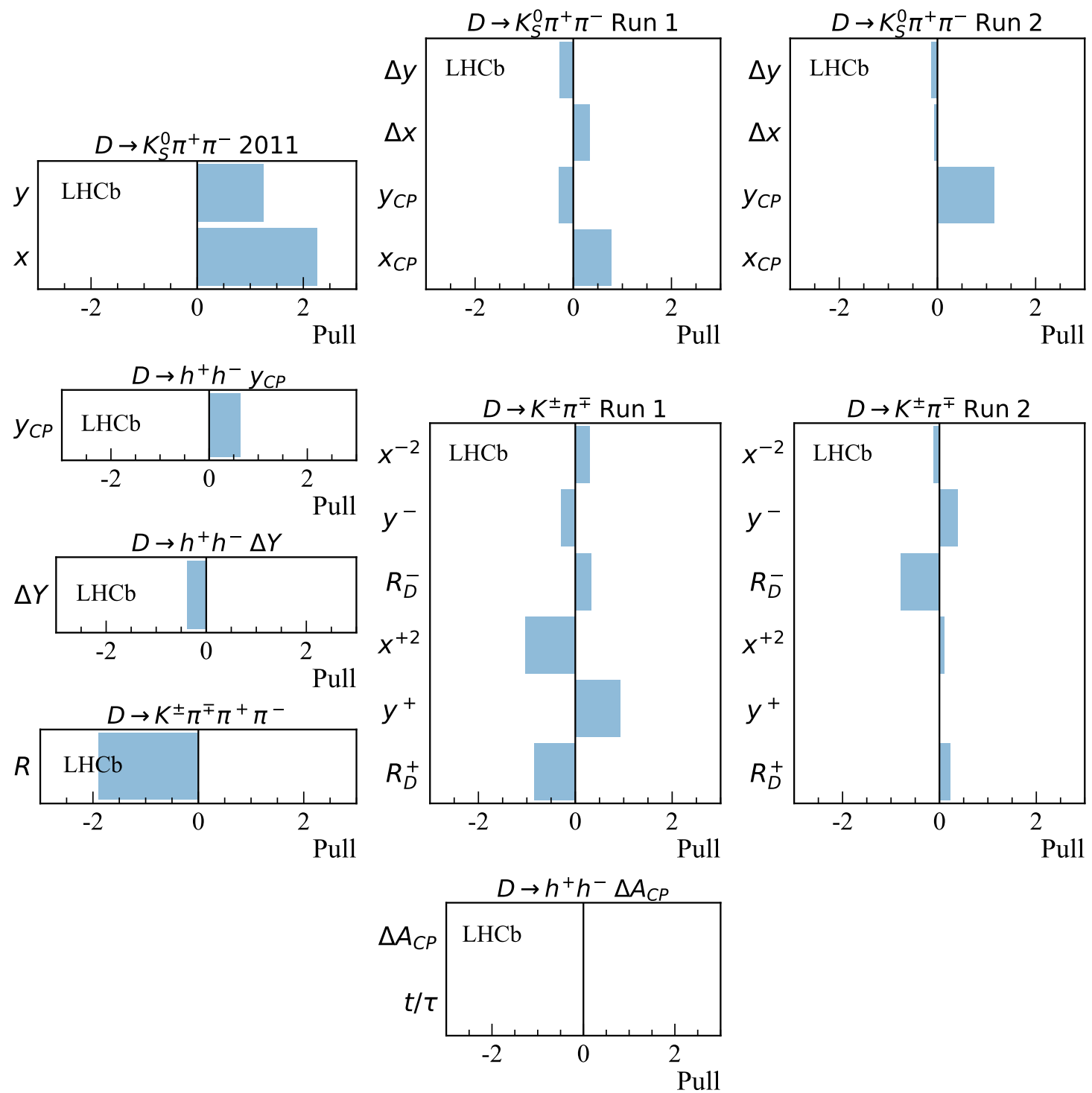

Figure 8. Pulls of the input observables, part 3 of 3. 


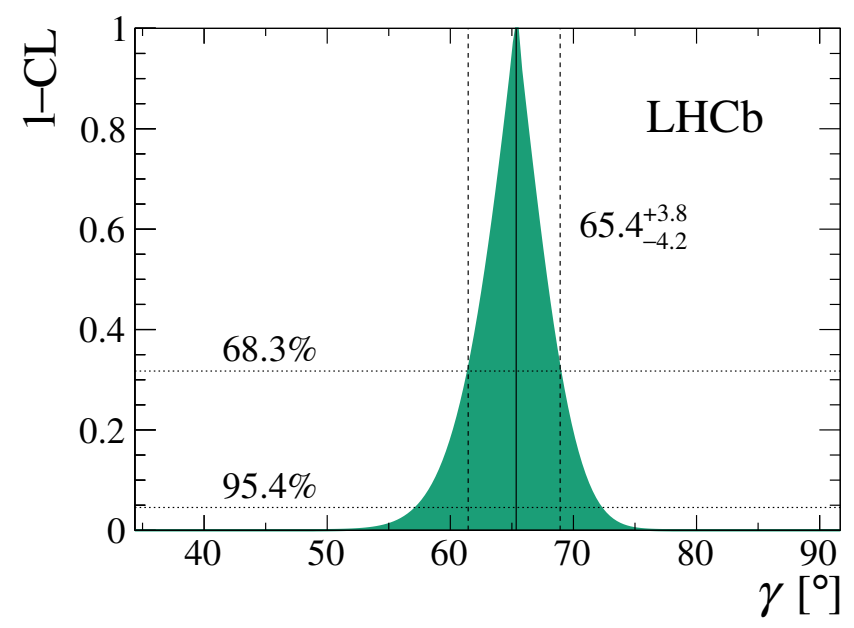

Figure 9. One dimensional 1 - CL profile for $\gamma$ from all inputs used in the combination.

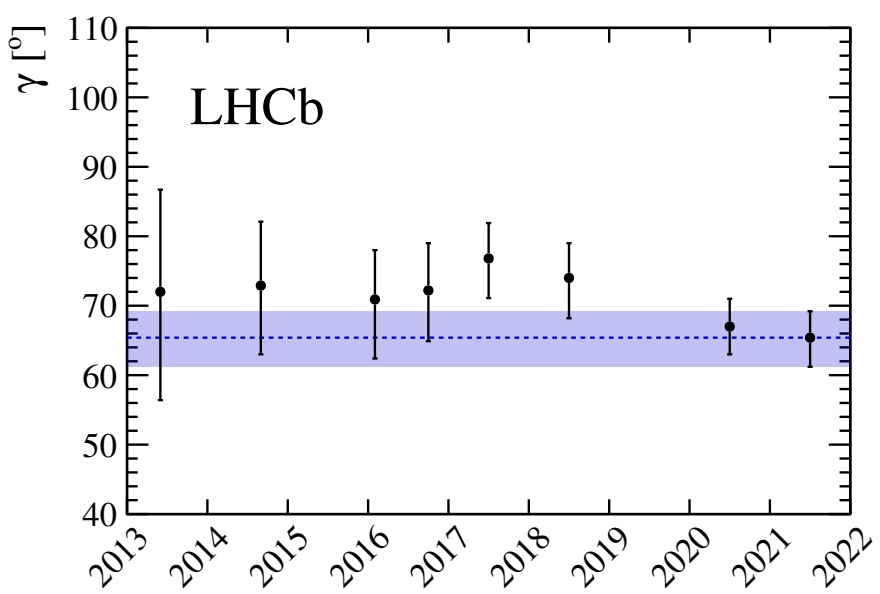

Figure 10. Evolution of the $\mathrm{LHCb}$ combination result for $\gamma$, with the central values and $1 \sigma$ uncertainties in black. This result is the 2021 data point, the value and uncertainty are highlighted by the dashed blue line and band, respectively.

\section{Additional figures}

Figure 9 shows the $p$-value distribution as a function of $\gamma$ for the global fit. A summary of $\mathrm{LHCb} \gamma$ combination results as a function of time is given in figure 10 .

Open Access. This article is distributed under the terms of the Creative Commons Attribution License (CC-BY 4.0), which permits any use, distribution and reproduction in any medium, provided the original author(s) and source are credited. 


\section{References}

[1] M. Gronau and D. Wyler, On determining a weak phase from CP asymmetries in charged B decays, Phys. Lett. B 265 (1991) 172 [INSPIRE].

[2] M. Gronau and D. London, How to determine all the angles of the unitarity triangle from $B_{d}^{0} \rightarrow D K_{s}$ and $B_{s}^{0} \rightarrow D^{0}$, Phys. Lett. B 253 (1991) 483 [INSPIRE].

[3] D. Atwood, I. Dunietz and A. Soni, Enhanced CP-violation with $B \rightarrow K D^{0}\left(\bar{D}^{0}\right)$ modes and extraction of the CKM angle $\gamma$, Phys. Rev. Lett. 78 (1997) 3257 [hep-ph/9612433] [INSPIRE].

[4] D. Atwood, I. Dunietz and A. Soni, Improved methods for observing CP-violation in $B^{ \pm} \rightarrow K D$ and measuring the CKM phase $\gamma$, Phys. Rev. D 63 (2001) 036005 [hep-ph/0008090] [INSPIRE].

[5] A. Bondar, Proceedings of BINP special analysis meeting on Dalitz analysis, unpublished, 24-26 September 2002.

[6] A. Giri, Y. Grossman, A. Soffer and J. Zupan, Determining $\gamma$ using $B^{ \pm} \rightarrow D K^{ \pm}$with multibody D decays, Phys. Rev. D 68 (2003) 054018 [hep-ph/0303187] [INSPIRE].

[7] BeLle collaboration, Measurement of $\phi_{3}$ with Dalitz plot analysis of $B^{ \pm} \rightarrow D^{(*)} K^{ \pm}$decay, Phys. Rev. D 70 (2004) 072003 [hep-ex/0406067] [InSPIRE].

[8] Y. Grossman, Z. Ligeti and A. Soffer, Measuring $\gamma$ in $B^{ \pm} \rightarrow K^{ \pm}\left(K K^{*}\right)_{D}$ decays, Phys. Rev. D 67 (2003) 071301 [hep-ph/0210433] [INSPIRE].

[9] J. Brod and J. Zupan, The ultimate theoretical error on $\gamma$ from $B \rightarrow D K$ decays, JHEP 01 (2014) 051 [arXiv: 1308.5663] [INSPIRE].

[10] J. Brod, A. Lenz, G. Tetlalmatzi-Xolocotzi and M. Wiebusch, New physics effects in tree-level decays and the precision in the determination of the quark mixing angle $\gamma$, Phys. Rev. D 92 (2015) 033002 [arXiv: 1412.1446] [INSPIRE].

[11] HFLAV collaboration, Averages of b-hadron, c-hadron, and $\tau$-lepton properties as of 2018, Eur. Phys. J. C 81 (2021) 226 [arXiv: 1909.12524] [INSPIRE].

[12] J. Charles et al., Current status of the Standard Model CKM fit and constraints on $\Delta F=2$ new physics, Phys. Rev. D 91 (2015) 073007 [arXiv:1501.05013] [InSPIRE].

[13] UTFIT collaboration, The unitarity triangle fit in the standard model and hadronic parameters from lattice QCD: a reappraisal after the measurements of $\Delta m_{s}$ and $B R\left(B \rightarrow \tau \nu_{\tau}\right)$, JHEP 10 (2006) 081 [hep-ph/0606167] [INSPIRE].

[14] M. Rama, Effect of $D-\bar{D}$ mixing in the extraction of gamma with $B^{-} \rightarrow D^{0} K^{-}$and $B^{-} \rightarrow D^{0} \pi^{-}$decays, Phys. Rev. D 89 (2014) 014021 [arXiv: 1307.4384] [InSPIRE].

[15] LHCb collaboration, Measurement of the CKM angle $\gamma$ from a combination of LHCb results, JHEP 12 (2016) 087 [arXiv:1611.03076] [INSPIRE].

[16] LHCb collaboration, Measurement of the CKM angle $\gamma$ from a combination of $B^{ \pm} \rightarrow D h^{ \pm}$ analyses, Phys. Lett. B 726 (2013) 151 [arXiv:1305.2050] [INSPIRE].

[17] LHCb collaboration, Update of the LHCb combination of the CKM angle $\gamma$, Tech. Rep. LHCb-CONF-2018-002, CERN, Geneva, Switzerland (2018) [CERN-LHCb-CONF-2018-002].

[18] LHCb collaboration, Updated LHCb combination of the CKM angle $\gamma$, Tech. Rep. LHCb-CONF-2020-003, CERN, Geneva, Switzerland (2020) [CERN-LHCb-CONF-2020-003]. 
[19] LHCb collaboration, Measurement of the CKM angle $\gamma$ in $B^{ \pm} \rightarrow D K^{ \pm}$and $B^{ \pm} \rightarrow D \pi^{ \pm}$ decays with $D \rightarrow K_{\mathrm{S}}^{0} h^{+} h^{-}$, JHEP 02 (2021) 169 [arXiv:2010.08483] [INSPIRE].

[20] LHCb collaboration, Measurement of CP observables in $B^{ \pm} \rightarrow D^{(*)} K^{ \pm}$and $B^{ \pm} \rightarrow D^{(*)} \pi^{ \pm}$ decays using two-body D final states, JHEP 04 (2021) 081 [arXiv:2012.09903] [INSPIRE].

[21] LHCb collaboration, Measurement of CP observables in $B^{ \pm} \rightarrow D K^{ \pm}$and $B^{ \pm} \rightarrow D \pi^{ \pm}$with two- and four-body D decays, Phys. Lett. B $\mathbf{7 6 0}$ (2016) 117 [arXiv:1603.08993] [INSPIRE].

[22] LHCb collaboration, A study of CP violation in $B^{\mp} \rightarrow D h^{\mp}(h=K, \pi)$ with the modes $D \rightarrow K^{\mp} \pi^{ \pm} \pi^{0}, D \rightarrow \pi^{+} \pi^{-} \pi^{0}$ and $D \rightarrow K^{+} K^{-} \pi^{0}$, Phys. Rev. $D 91$ (2015) 112014 [arXiv: 1504.05442] [INSPIRE].

[23] LHCb collaboration, Measurement of CP observables in $B^{ \pm} \rightarrow D K^{ \pm}$and $B^{ \pm} \rightarrow D \pi^{ \pm}$with $D \rightarrow K_{\mathrm{S}}^{0} K^{ \pm} \pi^{\mp}$ decays, JHEP 06 (2020) 058 [arXiv: 2002.08858] [INSPIRE].

[24] LHCb collaboration, Measurement of CP observables in $B^{ \pm} \rightarrow D K^{* \pm}$ decays using two- and four-body D final states, JHEP 11 (2017) 156 [Erratum ibid. 05 (2018) 067] [arXiv: 1709.05855] [INSPIRE].

[25] LHCb collaboration, Study of $B^{-} \rightarrow D K^{-} \pi^{+} \pi^{-}$and $B^{-} \rightarrow D \pi^{-} \pi^{+} \pi^{-}$decays and determination of the CKM angle $\gamma$, Phys. Rev. D 92 (2015) 112005 [arXiv:1505.07044] [INSPIRE].

[26] LHCb collaboration, Measurement of $C P$ observables in the process $B^{0} \rightarrow D K^{* 0}$ with twoand four-body D decays, JHEP 08 (2019) 041 [arXiv: 1906. 08297] [INSPIRE].

[27] LHCb collaboration, Measurement of the CKM angle $\gamma$ using $B^{0} \rightarrow D K^{* 0}$ with $D \rightarrow K_{S}^{0} \pi^{+} \pi^{-}$decays, JHEP 08 (2016) 137 [arXiv: 1605.01082] [INSPIRE].

[28] LHCb collaboration, Measurement of CP violation in $B^{0} \rightarrow D^{\mp} \pi^{ \pm}$decays, JHEP 06 (2018) 084 [arXiv: 1805.03448] [INSPIRE].

[29] LHCb collaboration, Measurement of CP asymmetry in $B_{s}^{0} \rightarrow D_{s}^{\mp} K^{ \pm}$decays, JHEP 03 (2018) 059 [arXiv : 1712.07428] [InSPIRE].

[30] LHCb collaboration, Measurement of the CKM angle $\gamma$ and $B_{s}^{0}-\bar{B}_{s}^{0}$ mixing frequency with $B_{s}^{0} \rightarrow D_{s}^{\mp} h^{ \pm} \pi^{ \pm} \pi^{\mp}$ decays, JHEP 03 (2021) 137 [arXiv:2011.12041] [INSPIRE].

[31] LHCb collaboration, Observation of CP-violation in charm decays, Phys. Rev. Lett. 122 (2019) 211803 [arXiv:1903.08726] [INSPIRE].

[32] LHCb collaboration, Measurement of CP asymmetry in $D^{0} \rightarrow K^{-} K^{+}$and $D^{0} \rightarrow \pi^{-} \pi^{+}$ decays, JHEP 07 (2014) 041 [arXiv: 1405.2797] [INSPIRE].

[33] LHCb collaboration, Measurement of the difference of time-integrated CP asymmetries in $D^{0} \rightarrow K^{-} K^{+}$and $D^{0} \rightarrow \pi^{-} \pi^{+}$decays, Phys. Rev. Lett. 116 (2016) 191601 [arXiv: 1602.03160] [INSPIRE].

[34] LHCb collaboration, Measurement of the charm-mixing parameter $y_{\mathrm{CP}}$, Phys. Rev. Lett. 122 (2019) 011802 [arXiv:1810.06874] [INSPIRE].

[35] LHCb collaboration, Measurement of indirect $C P$ asymmetries in $D^{0} \rightarrow K^{-} K^{+}$and $D^{0} \rightarrow \pi^{-} \pi^{+}$decays using semileptonic $B$ decays, JHEP 04 (2015) 043 [arXiv:1501.06777] [INSPIRE].

[36] LHCb collaboration, Measurement of the CP violation parameter $A_{\Gamma}$ in $D^{0} \rightarrow K^{+} K^{-}$and $D^{0} \rightarrow \pi^{+} \pi^{-}$decays, Phys. Rev. Lett. 118 (2017) 261803 [arXiv:1702.06490] [INSPIRE]. 
[37] LHCb collaboration, Updated measurement of decay-time-dependent CP asymmetries in $D^{0} \rightarrow K^{+} K^{-}$and $D^{0} \rightarrow \pi^{+} \pi^{-}$decays, Phys. Rev. D 101 (2020) 012005

[arXiv: 1911.01114] [INSPIRE].

[38] LHCb collaboration, Search for time-dependent CP violation in $D^{0} \rightarrow K^{+} K^{-}$and $D^{0} \rightarrow \pi^{+} \pi^{-}$decays, Phys. Rev. D 104 (2021) 072010 [arXiv:2105.09889] [inSPIRE].

[39] LHCb collaboration, Measurements of charm mixing and CP violation using $D^{0} \rightarrow K^{ \pm} \pi^{\mp}$ decays, Phys. Rev. D 95 (2017) 052004 [Erratum ibid. 96 (2017) 099907] [arXiv: 1611.06143] [INSPIRE].

[40] LHCb collaboration, Updated determination of $D^{0}-\bar{D}^{0}$ mixing and CP-violation parameters with $D^{0} \rightarrow K^{+} \pi^{-}$decays, Phys. Rev. D 97 (2018) 031101 [arXiv:1712.03220] [INSPIRE].

[41] LHCb collaboration, First observation of $D^{0}-\bar{D}^{0}$ oscillations in $D^{0} \rightarrow K^{+} \pi^{-} \pi^{+} \pi^{-}$decays and measurement of the associated coherence parameters, Phys. Rev. Lett. 116 (2016) 241801 [arXiv: 1602.07224] [INSPIRE].

[42] LHCb collaboration, Model-independent measurement of mixing parameters in $D^{0} \rightarrow K_{S}^{0} \pi^{+} \pi^{-}$decays, JHEP 04 (2016) 033 [arXiv: 1510.01664] [INSPIRE].

[43] LHCb collaboration, Measurement of the mass difference between neutral charm-meson eigenstates, Phys. Rev. Lett. 122 (2019) 231802 [arXiv: 1903.03074] [INSPIRE].

[44] LHCb collaboration, Observation of the mass difference between neutral charm-meson eigenstates, Phys. Rev. Lett. 127 (2021) 111801 [arXiv:2106.03744] [INSPIRE].

[45] LHCb collaboration, Constraints on the unitarity triangle angle $\gamma$ from Dalitz plot analysis of $B^{0} \rightarrow D K^{+} \pi^{-}$decays, Phys. Rev. D 93 (2016) 112018 [Erratum ibid. 94 (2016) 079902] [arXiv: 1602.03455] [INSPIRE].

[46] S. Malde et al., First determination of the CP content of $D \rightarrow \pi^{+} \pi^{-} \pi^{+} \pi^{-}$and updated determination of the $C P$ contents of $D \rightarrow \pi^{+} \pi^{-} \pi^{0}$ and $D \rightarrow K^{+} K^{-} \pi^{0}$, Phys. Lett. $B 747$ (2015) 9 [arXiv: 1504.05878] [INSPIRE].

[47] J. Libby et al., New determination of the $D^{0} \rightarrow K^{-} \pi^{+} \pi^{0}$ and $D^{0} \rightarrow K^{-} \pi^{+} \pi^{+} \pi^{-}$coherence factors and average strong-phase differences, Phys. Lett. B 731 (2014) 197 [arXiv: 1401.1904] [INSPIRE].

[48] T. Evans, S. Harnew, J. Libby, S. Malde, J. Rademacker and G. Wilkinson, Improved determination of the $D \rightarrow K^{-} \pi^{+} \pi^{+} \pi^{-}$coherence factor and associated hadronic parameters from a combination of $e^{+} e^{-} \rightarrow \psi(3770) \rightarrow c \bar{c}$ and $p p \rightarrow c \bar{c} X$ data, Phys. Lett. B 757 (2016) 520 [Erratum ibid. 765 (2017) 402] [arXiv: 1602 .07430] [InSPIRE].

[49] BESIII collaboration, Measurement of the $D \rightarrow K^{-} \pi^{+} \pi^{+} \pi^{-}$and $D \rightarrow K^{-} \pi^{+} \pi^{0}$ coherence factors and average strong-phase differences in quantum-correlated $D \bar{D}$ decays, JHEP 05 (2021) 164 [arXiv : 2103.05988] [INSPIRE].

[50] CLEO collaboration, Studies of the decays $D^{0} \rightarrow K_{S}^{0} K^{-} \pi^{+}$and $D^{0} \rightarrow K_{S}^{0} K^{+} \pi^{-}$, Phys. Rev. D 85 (2012) 092016 [Erratum ibid. 94 (2016) 099905] [arXiv: 1203.3804] [INSPIRE].

[51] LHCb collaboration, Studies of the resonance structure in $D^{0} \rightarrow K_{S}^{0} K^{ \pm} \pi^{\mp}$ decays, Phys. Rev. D 93 (2016) 052018 [arXiv: 1509.06628] [INSPIRE].

[52] M. Bjørn and S. Malde, CP violation and material interaction of neutral kaons in measurements of the CKM angle $\gamma$ using $B^{ \pm} \rightarrow D K^{ \pm}$decays where $D \rightarrow K_{S}^{0} \pi^{+} \pi^{-}$, JHEP 07 (2019) 106 [arXiv: 1904.01129] [INSPIRE]. 
[53] CLEO collaboration, Model-independent determination of the strong-phase difference between $D^{0}$ and $\bar{D}^{0} \rightarrow K_{S, L}^{0} h^{+} h^{-}(h=\pi, K)$ and its impact on the measurement of the CKM angle $\gamma / \phi_{3}$, Phys. Rev. D 82 (2010) 112006 [arXiv:1010.2817] [InSPIRE].

[54] BESIII collaboration, Model-independent determination of the relative strong-phase difference between $D^{0}$ and $\bar{D}^{0} \rightarrow K_{S, L}^{0} \pi^{+} \pi^{-}$and its impact on the measurement of the CKM angle $\gamma / \phi_{3}$, Phys. Rev. D 101 (2020) 112002 [arXiv:2003.00091] [INSPIRE].

[55] BESIII collaboration, Determination of strong-phase parameters in $D \rightarrow K_{S, L}^{0} \pi^{+} \pi^{-}$, Phys. Rev. Lett. 124 (2020) 241802 [arXiv:2002.12791] [INSPIRE].

[56] BESIII collaboration, Improved model-independent determination of the strong-phase difference between $D^{0}$ and $\bar{D}^{0} \rightarrow K_{\mathrm{S}, \mathrm{L}}^{0} K^{+} K^{-}$decays, Phys. Rev. D 102 (2020) 052008 [arXiv:2007.07959] [INSPIRE].

[57] B. Sen, M. Walker and M. Woodroofe, On the unified method with nuisance parameters, Statist. Sinica 19 (2009) 301.

[58] CLEO collaboration, Updated measurement of the strong phase in $D^{0} \rightarrow K^{+} \pi^{-}$decay using quantum correlations in $e^{+} e^{-} \rightarrow D^{0} \bar{D}^{0}$ at CLEO, Phys. Rev. D 86 (2012) 112001 [arXiv: 1210.0939] [INSPIRE].

[59] BESIII collaboration, Measurement of the $D \rightarrow K^{-} \pi^{+}$strong phase difference in $\psi(3770) \rightarrow D^{0} \bar{D}^{0}$, Phys. Lett. B 734 (2014) 227 [arXiv:1404.4691] [InSPIRE].

[60] T. Pajero and M.J. Morello, Mixing and $C P$ violation in $D^{0} \rightarrow K^{-} \pi^{+}$decays, arXiv: 2106.02014 [INSPIRE]. 


\section{The LHCb collaboration}

R. Aaij ${ }^{32}$, A.S.W. Abdelmotteleb ${ }^{56}$, C. Abellán Beteta ${ }^{50}$, F. Abudinén ${ }^{56}$, T. Ackernley ${ }^{60}$, B. Adeva ${ }^{46}$, M. Adinolfi ${ }^{54}$, H. Afsharnia ${ }^{9}$, C. Agapopoulou ${ }^{13}$, C.A. Aidala ${ }^{87}$, S. Aiola ${ }^{25}$, Z. Ajaltouni ${ }^{9}$, S. Akar ${ }^{65}$, J. Albrecht ${ }^{15}$, F. Alessio ${ }^{48}$, M. Alexander ${ }^{59}$, A. Alfonso Albero ${ }^{45}$, Z. Aliouche ${ }^{62}$, G. Alkhazov ${ }^{38}$, P. Alvarez Cartelle ${ }^{55}$, S. Amato ${ }^{2}$, J.L. Amey ${ }^{54}$, Y. Amhis ${ }^{11}$, L. $\mathrm{An}^{48}$, L. Anderlini ${ }^{22}$, A. Andreianov ${ }^{38}$, M. Andreotti ${ }^{21}$, F. Archilli ${ }^{17}$, A. Artamonov ${ }^{44}$, M. Artuso ${ }^{68}$, K. Arzymatov ${ }^{42}$, E. Aslanides ${ }^{10}$, M. Atzeni ${ }^{50}$, B. Audurier ${ }^{12}$, S. Bachmann ${ }^{17}$, M. Bachmayer ${ }^{49}$, J.J. Back ${ }^{56}$, P. Baladron Rodriguez ${ }^{46}$, V. Balagura ${ }^{12}$, W. Baldini ${ }^{21}$, J. Baptista Leite ${ }^{1}$, M. Barbetti ${ }^{22, g}$, R.J. Barlow ${ }^{62}$, S. Barsuk ${ }^{11}$, W. Barter ${ }^{61}$, M. Bartolini ${ }^{24, h}$, F. Baryshnikov ${ }^{83}$, J.M. Basels ${ }^{14}$, S. Bashir ${ }^{34}$, G. Bassi ${ }^{29}$, B. Batsukh ${ }^{68}$, A. Battig ${ }^{15}$, A. Bay ${ }^{49}$, A. Beck ${ }^{56}$, M. Becker ${ }^{15}$, F. Bedeschi ${ }^{29}$, I. Bediaga ${ }^{1}$, A. Beiter ${ }^{68}$, V. Belavin ${ }^{42}$, S. Belin ${ }^{27}$, V. Bellee ${ }^{50}$, K. Belous ${ }^{44}$, I. Belov ${ }^{40}$, I. Belyaev ${ }^{41}$, G. Bencivenni ${ }^{23}$, E. Ben-Haim ${ }^{13}$, A. Berezhnoy ${ }^{40}$, R. Bernet ${ }^{50}$, D. Berninghoff ${ }^{17}$, H.C. Bernstein ${ }^{68}$, C. Bertella ${ }^{48}$, A. Bertolin ${ }^{28}$, C. Betancourt ${ }^{50}$, F. Betti ${ }^{48}$, Ia. Bezshyiko ${ }^{50}$, S. Bhasin ${ }^{54}$, J. Bhom ${ }^{35}$, L. Bian ${ }^{73}$, M.S. Bieker ${ }^{15}$, S. Bifani ${ }^{53}$, P. Billoir ${ }^{13}$, M. Birch ${ }^{61}$, F.C.R. Bishop ${ }^{55}$, A. Bitadze ${ }^{62}$, A. Bizzeti ${ }^{22, k}$, M. Bjørn ${ }^{63}$, M.P. Blago ${ }^{48}$, T. Blake ${ }^{56}$, F. Blanc ${ }^{49}$, S. Blusk ${ }^{68}$, D. Bobulska ${ }^{59}$, J.A. Boelhauve ${ }^{15}$, O. Boente Garcia ${ }^{46}$, T. Boettcher ${ }^{65}$, A. Boldyrev ${ }^{82}$, A. Bondar ${ }^{43}$, N. Bondar ${ }^{38,48}$, S. Borghi ${ }^{62}$, M. Borisyak ${ }^{42}$, M. Borsato ${ }^{17}$, J.T. Borsuk ${ }^{35}$, S.A. Bouchiba ${ }^{49}$, T.J.V. Bowcock ${ }^{60}$, A. Boyer ${ }^{48}$, C. Bozzi ${ }^{21}$, M.J. Bradley ${ }^{61}$, S. Braun ${ }^{66}$, A. Brea Rodriguez ${ }^{46}$, J. Brodzicka ${ }^{35}$,

A. Brossa Gonzalo ${ }^{56}$, D. Brundu ${ }^{27}$, A. Buonaura ${ }^{50}$, L. Buonincontri ${ }^{28}$, A.T. Burke ${ }^{62}$, C. Burr ${ }^{48}$, A. Bursche ${ }^{72}$, A. Butkevich ${ }^{39}$, J.S. Butter ${ }^{32}$, J. Buytaert ${ }^{48}$, W. Byczynski ${ }^{48}$, S. Cadeddu ${ }^{27}$, H. Cai ${ }^{73}$, R. Calabrese ${ }^{21, f}$, L. Calefice ${ }^{15,13}$, L. Calero Diaz ${ }^{23}$, S. Cali ${ }^{23}$, R. Calladine ${ }^{53}$, M. Calvi ${ }^{26, j}$, M. Calvo Gomez ${ }^{85}$, P. Camargo Magalhaes ${ }^{54}$, P. Campana ${ }^{23}$, A.F. Campoverde Quezada ${ }^{6}$, S. Capelli ${ }^{26, j}$, L. Capriotti $^{20, d}$, A. Carbone ${ }^{20, d}$, G. Carboni ${ }^{31}$, R. Cardinale ${ }^{24, h}$, A. Cardini ${ }^{27}$, I. Carli ${ }^{4}$, P. Carniti ${ }^{26, j}$, L. Carus ${ }^{14}$, K. Carvalho Akiba ${ }^{32}$, A. Casais Vidal ${ }^{46}$, G. Casse ${ }^{60}$, M. Cattaneo ${ }^{48}$, G. Cavallero ${ }^{48}$, S. Celani $^{49}$, J. Cerasoli ${ }^{10}$, D. Cervenkov ${ }^{63}$, A.J. Chadwick ${ }^{60}$, M.G. Chapman ${ }^{54}$, M. Charles ${ }^{13}$, Ph. Charpentier ${ }^{48}$, G. Chatzikonstantinidis ${ }^{53}$, C.A. Chavez Barajas ${ }^{60}$, M. Chefdeville ${ }^{8}$, C. Chen $^{3}$, S. Chen ${ }^{4}$, A. Chernov ${ }^{35}$, V. Chobanova ${ }^{46}, \mathrm{~S} . \mathrm{Cholak}^{49}, \mathrm{M} . \mathrm{Chrzaszcz}^{35}, \mathrm{~A} \cdot \mathrm{Chubykin}^{38}$, V. Chulikov ${ }^{38}$, P. Ciambrone ${ }^{23}$, M.F. Cicala ${ }^{56}$, X. Cid Vidal ${ }^{46}$, G. Ciezarek $^{48}$, P.E.L. Clarke ${ }^{58}$, M. Clemencic ${ }^{48}$, H.V. Cliff ${ }^{55}$, J. Closier ${ }^{48}$, J.L. Cobbledick ${ }^{62}$, V. Coco $^{48}$, J.A.B. Coelho ${ }^{11}$, J. Cogan ${ }^{10}$,

E. Cogneras $^{9}$, L. Cojocariu ${ }^{37}$, P. Collins ${ }^{48}$, T. Colombo ${ }^{48}$, L. Congedo ${ }^{19, c}$, A. Contu ${ }^{27}$, N. Cooke ${ }^{53}$, G. Coombs $^{59}$, I. Corredoira ${ }^{46}$, G. Corti ${ }^{48}$, C.M. Costa Sobral ${ }^{56}$, B. Couturier ${ }^{48}$, D.C. Craik ${ }^{64}$, J. Crkovská ${ }^{67}$, M. Cruz Torres ${ }^{1}$, R. Currie $^{58}$, C.L. Da Silva ${ }^{67}$, S. Dadabaev ${ }^{83}$, L. Dai ${ }^{71}$, E. Dall'Occo ${ }^{15}$, J. Dalseno ${ }^{46}$, C. D'Ambrosio ${ }^{48}$, A. Danilina ${ }^{41}$, P. d'Argent ${ }^{48}$, J.E. Davies ${ }^{62}$, A. Davis ${ }^{62}$, O. De Aguiar Francisco ${ }^{62}$, K. De Bruyn ${ }^{79}$, S. De Capua ${ }^{62}$, M. De Cian ${ }^{49}$, J.M. De Miranda ${ }^{1}$, L. De Paula ${ }^{2}$, M. De Serio ${ }^{19, c}$, D. De Simone ${ }^{50}$, P. De Simone ${ }^{23}$, F. De Vellis ${ }^{15}$, J.A. de Vries ${ }^{80}$, C.T. Dean ${ }^{67}$, F. Debernardis ${ }^{19, c}$, D. Decamp ${ }^{8}$, V. Dedu ${ }^{10}$, L. Del Buono ${ }^{13}$, B. Delaney ${ }^{55}$, H.-P. Dembinski ${ }^{15}$, A. Dendek ${ }^{34}$, V. Denysenko ${ }^{50}$, D. Derkach ${ }^{82}$, O. Deschamps ${ }^{9}$, F. Desse ${ }^{11}$, F. Dettori ${ }^{27, e}$, B. Dey ${ }^{77}$, A. Di Cicco ${ }^{23}$, P. Di Nezza ${ }^{23}$, S. Didenko ${ }^{83}$, L. Dieste Maronas ${ }^{46}$, H. Dijkstra ${ }^{48}$, V. Dobishuk ${ }^{52}$, C. Dong ${ }^{3}$, A.M. Donohoe ${ }^{18}$, F. Dordei ${ }^{27}$, A.C. dos Reis ${ }^{1}$, L. Douglas ${ }^{59}$, A. Dovbnya ${ }^{51}$, A.G. Downes ${ }^{8}$, M.W. Dudek ${ }^{35}$, L. Dufour ${ }^{48}$, V. Duk ${ }^{78}$, P. Durante ${ }^{48}$, J.M. Durham ${ }^{67}$, D. Dutta ${ }^{62}$, A. Dziurda ${ }^{35}$, A. Dzyuba ${ }^{38}$, S. Easo ${ }^{57}$, U. Egede ${ }^{69}$, V. Egorychev ${ }^{41}$, S. Eidelman ${ }^{43, u, \dagger}$, S. Eisenhardt ${ }^{58}$, S. Ek-In ${ }^{49}$, L. Eklund ${ }^{59,86}$, S. Ely ${ }^{68}$, A. Ene ${ }^{37}$, E. Epple ${ }^{67}$, S. Escher ${ }^{14}$, J. Eschle ${ }^{50}$, S. Esen ${ }^{13}$, T. Evans ${ }^{48}$,

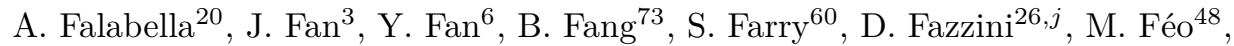

A. Fernandez Prieto ${ }^{46}$, A.D. Fernez ${ }^{66}$, F. Ferrari ${ }^{20, d}$, L. Ferreira Lopes ${ }^{49}$, F. Ferreira Rodrigues ${ }^{2}$, 
S. Ferreres Sole ${ }^{32}$, M. Ferrillo ${ }^{50}$, M. Ferro-Luzzi ${ }^{48}$, S. Filippov ${ }^{39}$, R.A. Fini ${ }^{19}$, M. Fiorini ${ }^{21, f}$, M. Firlej ${ }^{34}$, K.M. Fischer ${ }^{63}$, D.S. Fitzgerald ${ }^{87}$, C. Fitzpatrick ${ }^{62}$, T. Fiutowski ${ }^{34}$, A. Fkiaras ${ }^{48}$, F. Fleuret ${ }^{12}$, M. Fontana ${ }^{13}$, F. Fontanelli ${ }^{24, h}$, R. Forty ${ }^{48}$, D. Foulds-Holt ${ }^{55}$, V. Franco Lima ${ }^{60}$, M. Franco Sevilla ${ }^{66}$, M. Frank ${ }^{48}$, E. Franzoso ${ }^{21}$, G. Frau ${ }^{17}$, C. Frei ${ }^{48}$, D.A. Friday ${ }^{59}$, J. Fu ${ }^{6}$, Q. Fuehring ${ }^{15}$, E. Gabriel ${ }^{32}$, G. Galati ${ }^{19, c}$, A. Gallas Torreira ${ }^{46}$, D. Galli ${ }^{20, d}$, S. Gambetta ${ }^{58,48}$, Y. Gan ${ }^{3}$, M. Gandelman ${ }^{2}$, P. Gandini' ${ }^{25}$, Y. Gao ${ }^{5}$, M. Garau ${ }^{27}$, L.M. Garcia Martin ${ }^{56}$, P. Garcia Moreno ${ }^{45}$, J. García Pardiñas ${ }^{26, j}$, B. Garcia Plana ${ }^{46}$, F.A. Garcia Rosales ${ }^{12}$, L. Garrido ${ }^{45}$, C. Gasparr ${ }^{48}$, R.E. Geertsema ${ }^{32}$, D. Gerick ${ }^{17}$, L.L. Gerken ${ }^{15}$, E. Gersabeck ${ }^{62}$, M. Gersabeck ${ }^{62}$, T. Gershon ${ }^{56}$, D. Gerstel ${ }^{10}$, L. Giambastiani ${ }^{28}$, V. Gibson ${ }^{55}$, H.K. Giemza ${ }^{36}$, A.L. Gilman ${ }^{63}$, M. Giovannetti ${ }^{23, p}$, A. Gioventù ${ }^{46}$, P. Gironella Gironell ${ }^{45}$, L. Giubega ${ }^{37}$, C. Giugliano ${ }^{21, f, 48}$, K. Gizdov ${ }^{58}$, E.L. Gkougkousis ${ }^{48}$, V.V. Gligorov ${ }^{13}$, C. Göbel ${ }^{70}$, E. Golobardes ${ }^{85}$, D. Golubkov ${ }^{41}$, A. Golutvin ${ }^{61,83}$, A. Gomes ${ }^{1, a}$, S. Gomez Fernandez ${ }^{45}$, F. Goncalves Abrantes ${ }^{63}$, M. Goncerz ${ }^{35}$, G. Gong ${ }^{3}$, P. Gorbounov ${ }^{41}$, I.V. Gorelov ${ }^{40}$, C. Gotti ${ }^{26}$, E. Govorkova ${ }^{48}$, J.P. Grabowski ${ }^{17}$, T. Grammatico ${ }^{13}$, L.A. Granado Cardoso ${ }^{48}$, E. Graugés ${ }^{45}$, E. Graverini ${ }^{49}$, G. Graziani ${ }^{22}$, A. Grecu ${ }^{37}$, L.M. Greeven ${ }^{32}$, N.A. Grieser ${ }^{4}$, L. Grillo ${ }^{62}$, S. Gromov ${ }^{83}$, B.R. Gruberg Cazon $^{63}$, C. Gu ${ }^{3}$, M. Guarise ${ }^{21}$, M. Guittiere ${ }^{11}$, P. A. Günther ${ }^{17}$, E. Gushchin ${ }^{39}$, A. Guth ${ }^{14}$, Y. Guz ${ }^{44}$, T. Gys ${ }^{48}$, T. Hadavizadeh ${ }^{69}$, G. Haefeli ${ }^{49}$, C. Haen ${ }^{48}$, J. Haimberger ${ }^{48}$, T. Halewood-leagas ${ }^{60}$, P.M. Hamilton ${ }^{66}$, J.P. Hammerich ${ }^{60}$, Q. $\operatorname{Han}^{7}$, X. $\operatorname{Han}^{17}$, T.H. Hancock ${ }^{63}$, E.B. Hansen ${ }^{62}$, S. Hansmann-Menzemer ${ }^{17}$, N. Harnew ${ }^{63}$, T. Harrison ${ }^{60}$, C. Hasse ${ }^{48}$, M. Hatch ${ }^{48}$, J. He ${ }^{6, b}$, M. Hecker ${ }^{61}$, K. Heijhoff ${ }^{32}$, K. Heinicke ${ }^{15}$, A.M. Hennequin ${ }^{48}$, K. Hennessy ${ }^{60}$, L. Henry ${ }^{48}$, J. Heuel ${ }^{14}$, A. Hicheur ${ }^{2}$, D. Hill ${ }^{49}$, M. Hilton ${ }^{62}$, S.E. Hollitt ${ }^{15}$,

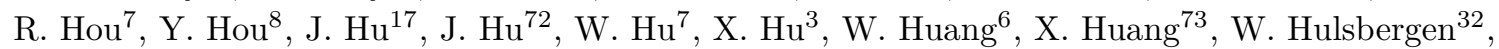
R.J. Hunter ${ }^{56}$, M. Hushchyn ${ }^{82}$, D. Hutchcroft ${ }^{60}$, D. $\operatorname{Hynds}^{32}$, P. Ibis ${ }^{15}$, M. Idzik ${ }^{34}$, D. Ilin ${ }^{38}$, P. Ilten ${ }^{65}$, A. Inglessi ${ }^{38}$, A. Ishteev ${ }^{83}$, K. Ivshin ${ }^{38}$, R. Jacobsson ${ }^{48}$, H. Jage ${ }^{14}$, S. Jakobsen ${ }^{48}$, E. Jans ${ }^{32}$, B.K. Jashal ${ }^{47}$, A. Jawahery ${ }^{66}$, V. Jevtic ${ }^{15}$, F. Jiang ${ }^{3}$, M. John ${ }^{63}$, D. Johnson ${ }^{48}$, C.R. Jones ${ }^{55}$, T.P. Jones ${ }^{56}$, B. Jost ${ }^{48}$, N. Jurik ${ }^{48}$, S.H. Kalavan Kadavath ${ }^{34}$, S. Kandybei ${ }^{51}$, Y. Kang ${ }^{3}$, M. Karacson ${ }^{48}$, M. Karpov ${ }^{82}$, F. Keizer ${ }^{48}$, D.M. Keller ${ }^{68}$, M. Kenzie ${ }^{56}$, T. Ketel ${ }^{33}$, B. Khanji ${ }^{15}$, A. Kharisova ${ }^{84}$, S. Kholodenko ${ }^{44}$, T. Kirn ${ }^{14}$, V.S. Kirsebom ${ }^{49}$, O. Kitouni ${ }^{64}$, S. Klaver ${ }^{32}$, N. Kleijne ${ }^{29}$, K. Klimaszewski ${ }^{36}$, M.R. Kmiec ${ }^{36}$, S. Koliiev ${ }^{52}$, A. Kondybayeva ${ }^{83}$, A. Konoplyannikov ${ }^{41}$, P. Kopciewicz ${ }^{34}$, R. Kopecna ${ }^{17}$, P. Koppenburg ${ }^{32}$, M. Korolev ${ }^{40}$, I. Kostiuk ${ }^{32,52}$, O. $\operatorname{Kot}^{52}$, S. Kotriakhova ${ }^{21,38}$, P. Kravchenko ${ }^{38}$, L. Kravchuk ${ }^{39}$, R.D. Krawczyk ${ }^{48}$, M. Kreps ${ }^{56}$, F. Kress ${ }^{61}$, S. Kretzschmar ${ }^{14}$, P. Krokovny ${ }^{43, u}$, W. Krupa ${ }^{34}$, W. Krzemien ${ }^{36}$, M. Kucharczyk ${ }^{35}$, V. Kudryavtsev ${ }^{43, u}$, H.S. Kuindersma ${ }^{32,33}$, G.J. Kunde ${ }^{67}$, T. Kvaratskheliya ${ }^{41}$,

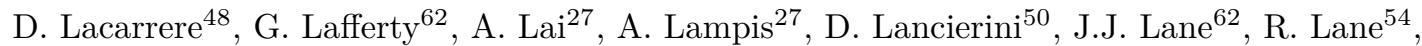
G. Lanfranchi ${ }^{23}$, C. Langenbruch ${ }^{14}$, J. Langer ${ }^{15}$, O. Lantwin ${ }^{83}$, T. Latham ${ }^{56}$, F. Lazzari ${ }^{29, q}$,

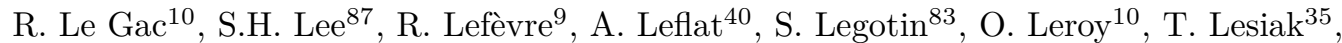
B. Leverington ${ }^{17}, \mathrm{H} . \mathrm{Li}^{72}, \mathrm{P} . \mathrm{Li}^{17}, \mathrm{~S} . \mathrm{Li}^{7}, \mathrm{Y} . \mathrm{Li}^{4}, \mathrm{Y} . \mathrm{Li}^{4}, \mathrm{Z} . \mathrm{Li}^{68}, \mathrm{X} . \mathrm{Liang}{ }^{68}, \mathrm{~T} . \mathrm{Lin}^{61}$, R. Lindner ${ }^{48}$, V. Lisovskyi ${ }^{15}$, R. Litvinov ${ }^{27}$, G. $\mathrm{Liu}^{72}, \mathrm{H} . \mathrm{Liu}^{6}$, Q. Liu ${ }^{6}$, S. Liu ${ }^{4}$, A. Lobo Salvia ${ }^{45}$, A. Loi ${ }^{27}$, J. Lomba Castro ${ }^{46}$, I. Longstaff ${ }^{59}$, J.H. Lopes ${ }^{2}$, S. Lopez Solino ${ }^{46}$, G.H. Lovell ${ }^{55}$, Y. Lu ${ }^{4}$, C. Lucarelli ${ }^{22, g}$, D. Lucchesi ${ }^{28, l}$, S. Luchuk ${ }^{39}$, M. Lucio Martinez ${ }^{32}$, V. Lukashenko ${ }^{32,52}$, Y. Luo ${ }^{3}$,

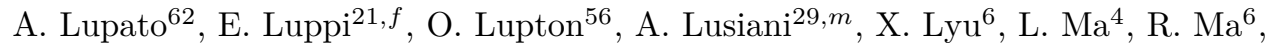

S. Maccolini ${ }^{20, d}$, F. Machefert ${ }^{11}$, F. Maciuc ${ }^{37}$, V. Macko ${ }^{49}$, P. Mackowiak ${ }^{15}$,

S. Maddrell-Mander ${ }^{54}$, O. Madejczyk ${ }^{34}$, L.R. Madhan Mohan ${ }^{54}$, O. Maev ${ }^{38}$, A. Maevskiy ${ }^{82}$, D. Maisuzenko ${ }^{38}$, M.W. Majewski ${ }^{34}$, J.J. Malczewski ${ }^{35}$, S. Malde ${ }^{63}$, B. Malecki ${ }^{48}$, A. Malinin ${ }^{81}$, T. Maltsev ${ }^{43, u}$, H. Malygina ${ }^{17}$, G. Manca ${ }^{27, e}$, G. Mancinelli ${ }^{10}$, D. Manuzzi ${ }^{20, d}$, D. Marangotto ${ }^{25, i}$, J. Maratas ${ }^{9, s}$, J.F. Marchand ${ }^{8}$, U. Marconi ${ }^{20}$, S. Mariani ${ }^{22, g}$, C. Marin Benito ${ }^{48}$, M. Marinangeli ${ }^{49}$, J. Marks ${ }^{17}$, A.M. Marshall ${ }^{54}$, P.J. Marshall ${ }^{60}$, G. Martelli ${ }^{78}$, G. Martellotti ${ }^{30}$, L. Martinazzoli ${ }^{48, j}$, M. Martinelli ${ }^{26, j}$, D. Martinez Santos ${ }^{46}$, F. Martinez Vidal ${ }^{47}$, A. Massafferri ${ }^{1}$, 


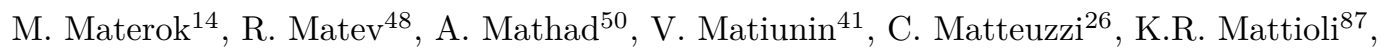
A. Mauri ${ }^{32}$, E. Maurice ${ }^{12}$, J. Mauricio ${ }^{45}$, M. Mazurek ${ }^{48}$, M. McCann ${ }^{61}$, L. Mcconnell $^{18}$, T.H. Mcgrath ${ }^{62}$, N.T. Mchugh ${ }^{59}$, A. McNab ${ }^{62}$, R. McNulty ${ }^{18}$, J.V. Mead ${ }^{60}$, B. Meadows ${ }^{65}$, G. Meier ${ }^{15}$, N. Meinert ${ }^{76}$, D. Melnychuk ${ }^{36}$, S. Meloni ${ }^{26, j}$, M. Merk ${ }^{32,80}$, A. Merli $^{25, i}$, L. Meyer Garcia ${ }^{2}$, M. Mikhasenko ${ }^{48}$, D.A. Milanes ${ }^{74}$, E. Millard ${ }^{56}$, M. Milovanovic ${ }^{48}$, M.-N. Minard ${ }^{8}$, A. Minotti ${ }^{26, j}$, L. Minzoni ${ }^{21, f}$, S.E. Mitchell ${ }^{58}$, B. Mitreska ${ }^{62}$, D.S. Mitzel ${ }^{15}$, A. Mödden ${ }^{15}$, R.A. Mohammed ${ }^{63}$, R.D. Moise ${ }^{61}$, S. Mokhnenko ${ }^{82}$, T. Mombächer ${ }^{46}$, I.A. Monroy ${ }^{74}$, S. Monteil ${ }^{9}$, M. Morandin ${ }^{28}$, G. Morello ${ }^{23}$, M.J. Morello ${ }^{29, m}$, J. Moron $^{34}$, A.B. Morris ${ }^{75}$, A.G. Morris ${ }^{56}$, R. Mountain ${ }^{68}, \mathrm{H} . \mathrm{Mu}^{3}$, F. Muheim ${ }^{58,48}$, M. Mulder ${ }^{48}$, D. Müller ${ }^{48}$, K. Müller ${ }^{50}$, C.H. Murphy ${ }^{63}$, D. Murray ${ }^{62}$, P. Muzzetto ${ }^{27,48}$, P. Naik ${ }^{54}$, T. Nakada ${ }^{49}$, R. Nandakumar ${ }^{57}$, T. Nanut ${ }^{49}$, I. Nasteva ${ }^{2}$, M. Needham ${ }^{58}$, I. Neri ${ }^{21}$, N. Neri ${ }^{25, i}$, S. Neubert ${ }^{75}$, N. Neufeld ${ }^{48}$, R. Newcombe ${ }^{61}$, E.M. Niel ${ }^{11}$, S. Nieswand ${ }^{14}$, N. Nikitin ${ }^{40}$, N.S. Nolte ${ }^{64}$, C. Normand ${ }^{8}$, C. Nunez ${ }^{87}$, A. Oblakowska-Mucha ${ }^{34}$, V. Obraztsov ${ }^{44}$, T. Oeser ${ }^{14}$, D.P. O'Hanlon ${ }^{54}$, S. Okamura ${ }^{21}$, R. Oldeman ${ }^{27, e}$, F. Oliva ${ }^{58}$, M.E. Olivares ${ }^{68}$, C.J.G. Onderwater ${ }^{79}$, R.H. O'Neil ${ }^{58}$, J.M. Otalora Goicochea ${ }^{2}$, T. Ovsiannikova ${ }^{41}$, P. Owen ${ }^{50}$, A. Oyanguren ${ }^{47}$, K.O. Padeken ${ }^{75}$, B. Pagare ${ }^{56}$, P.R. Pais ${ }^{48}$, T. Pajero ${ }^{63}$, A. Palano ${ }^{19}$,

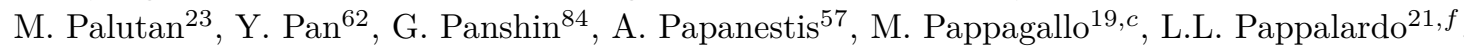
C. Pappenheimer ${ }^{65}$, W. Parker ${ }^{66}$, C. Parkes ${ }^{62}$, B. Passalacqua ${ }^{21}$, G. Passaleva ${ }^{22}$, A. Pastore ${ }^{19}$, M. Patel ${ }^{61}$, C. Patrignani ${ }^{20, d}$, C.J. Pawley ${ }^{80}$, A. Pearce ${ }^{48}$, A. Pellegrino ${ }^{32}$, M. Pepe Altarelli ${ }^{48}$,

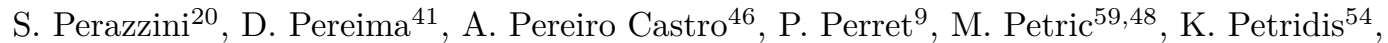

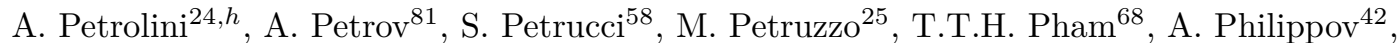
L. Pica ${ }^{29, m}$, M. Piccini ${ }^{78}$, B. Pietrzyk ${ }^{8}$, G. Pietrzyk ${ }^{49}$, M. Pili ${ }^{63}$, D. Pinci ${ }^{30}$, F. Pisani ${ }^{48}$, M. Pizzichemi ${ }^{26,48, j}$, Resmi P.K ${ }^{10}$, V. Placinta ${ }^{37}$, J. Plews ${ }^{53}$, M. Plo Casasus ${ }^{46}$, F. Polci ${ }^{13}$, M. Poli Lener ${ }^{23}$, M. Poliakova ${ }^{68}$, A. Poluektov ${ }^{10}$, N. Polukhina ${ }^{83, t}$, I. Polyakov ${ }^{68}$, E. Polycarpo ${ }^{2}$, S. Ponce ${ }^{48}$, D. Popov ${ }^{6,48}$, S. Popov ${ }^{42}$, S. Poslavskii ${ }^{44}$, K. Prasanth ${ }^{35}$, L. Promberger ${ }^{48}$, C. Prouve ${ }^{46}$, V. Pugatch ${ }^{52}$, V. Puill ${ }^{11}$, H. Pullen ${ }^{63}$, G. Punzi ${ }^{29, n}$, H. Qi ${ }^{3}$, W. Qian ${ }^{6}$, J. Qin ${ }^{6}$, N. Qin ${ }^{3}$, R. Quagliani ${ }^{49}$, B. Quintana ${ }^{8}$, N.V. Raab ${ }^{18}$, R.I. Rabadan Trejo ${ }^{6}$, B. Rachwal ${ }^{34}$, J.H. Rademacker ${ }^{54}$, M. Rama ${ }^{29}$, M. Ramos Pernas ${ }^{56}$, M.S. Rangel ${ }^{2}$, F. Ratnikov ${ }^{42,82}$, G. Raven ${ }^{33}$,

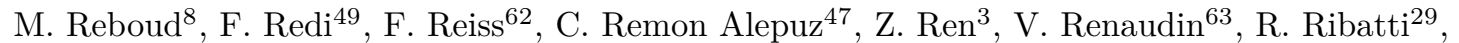
S. Ricciardi ${ }^{57}$, K. Rinnert ${ }^{60}$, P. Robbe ${ }^{11}$, G. Robertson ${ }^{58}$, A.B. Rodrigues ${ }^{49}$, E. Rodrigues $^{60}$, J.A. Rodriguez Lopez ${ }^{74}$, E.R.R. Rodriguez Rodriguez ${ }^{46}$, A. Rollings ${ }^{63}$, P. Roloff ${ }^{48}$, V. Romanovskiy ${ }^{44}$, M. Romero Lamas ${ }^{46}$, A. Romero Vidal ${ }^{46}$, J.D. Roth ${ }^{87}$, M. Rotondo ${ }^{23}$, M.S. Rudolph ${ }^{68}$, T. Ruf ${ }^{48}$, R.A. Ruiz Fernandez ${ }^{46}$, J. Ruiz Vidal ${ }^{47}$, A. Ryzhikov ${ }^{82}$, J. Ryzka ${ }^{34}$, J.J. Saborido Silva ${ }^{46}$, N. Sagidova ${ }^{38}$, N. Sahoo ${ }^{56}$, B. Saitta ${ }^{27, e}$, M. Salomoni ${ }^{48}$, C. Sanchez Gras ${ }^{32}$, R. Santacesaria ${ }^{30}$, C. Santamarina Rios ${ }^{46}$, M. Santimaria ${ }^{23}$, E. Santovetti ${ }^{31, p}$, D. Saranin ${ }^{83}$, G. Sarpis ${ }^{14}$, M. Sarpis ${ }^{75}$, A. Sarti ${ }^{30}$, C. Satriano ${ }^{30, o}$, A. Satta ${ }^{31}$, M. Saur ${ }^{15}$, D. Savrina ${ }^{41,40}$, H. Sazak ${ }^{9}$, L.G. Scantlebury Smead ${ }^{63}$, A. Scarabotto ${ }^{13}$, S. Schael ${ }^{14}$, S. Scherl ${ }^{60}$, M. Schiller ${ }^{59}$, H. Schindler ${ }^{48}$, M. Schmelling ${ }^{16}$, B. Schmidt ${ }^{48}$, S. Schmitt ${ }^{14}$, O. Schneider ${ }^{49}$, A. Schopper ${ }^{48}$, M. Schubiger ${ }^{32}$, S. Schulte ${ }^{49}$, M.H. Schune ${ }^{11}$, R. Schwemmer ${ }^{48}$, B. Sciascia ${ }^{23,48}$, S. Sellam ${ }^{46}$, A. Semennikov ${ }^{41}$, M. Senghi Soares ${ }^{33}$, A. Sergi ${ }^{24, h}$, N. Serra ${ }^{50}$, L. Sestini ${ }^{28}$, A. Seuthe ${ }^{15}$, Y. Shang ${ }^{5}$, D.M. Shangase ${ }^{87}$, M. Shapkin ${ }^{44}$, I. Shchemerov ${ }^{83}$, L. Shchutska ${ }^{49}$, T. Shears ${ }^{60}$, L. Shekhtman ${ }^{43, u}$, Z. Shen ${ }^{5}$, V. Shevchenko ${ }^{81}$, E.B. Shields ${ }^{26, j}$, Y. Shimizu ${ }^{11}$, E. Shmanin ${ }^{83}$, J.D. Shupperd ${ }^{68}$, B.G. Siddi ${ }^{21}$, R. Silva Coutinho ${ }^{50}$, G. Simi $^{28}$, S. Simone ${ }^{19, c}$, N. Skidmore ${ }^{62}$, T. Skwarnicki ${ }^{68}$, M.W. Slater ${ }^{53}$, I. Slazyk ${ }^{21, f}$, J.C. Smallwood ${ }^{63}$, J.G. Smeaton ${ }^{55}$, A. Smetkina ${ }^{41}$, E. Smith ${ }^{50}$, M. Smith ${ }^{61}$, A. Snoch ${ }^{32}$, M. Soares ${ }^{20}$, L. Soares Lavra ${ }^{9}$, M.D. Sokoloff ${ }^{65}$, F.J.P. Soler ${ }^{59}$, A. Solovev ${ }^{38}$, I. Solovyev ${ }^{38}$, F.L. Souza De Almeida ${ }^{2}$, B. Souza De Paula ${ }^{2}$, B. Spaan ${ }^{15}$, E. Spadaro Norella ${ }^{25, i}$, P. Spradlin ${ }^{59}$, F. Stagni ${ }^{48}$, M. Stahl ${ }^{65}$, S. Stahl ${ }^{48}$, S. Stanislaus ${ }^{63}$, O. Steinkamp ${ }^{50,83}$, O. Stenyakin ${ }^{44}$, H. Stevens ${ }^{15}$, S. Stone ${ }^{68}$, M. Straticiuc ${ }^{37}$, 
D. Strekalina ${ }^{83}$, F. Suljik ${ }^{63}$, J. Sun ${ }^{27}$, L. Sun ${ }^{73}$, Y. Sun ${ }^{66}$, P. Svihra ${ }^{62}$, P.N. Swallow ${ }^{53}$, K. Swientek ${ }^{34}$, A. Szabelski ${ }^{36}$, T. Szumlak ${ }^{34}$, M. Szymanski ${ }^{48}$, S. Taneja ${ }^{62}$, A.R. Tanner ${ }^{54}$, M.D. Tat $^{63}$, A. Terentev ${ }^{83}$, F. Teubert ${ }^{48}$, E. Thomas ${ }^{48}$, D.J.D. Thompson ${ }^{53}$, K.A. Thomson ${ }^{60}$, V. Tisserand ${ }^{9}$, S. T'Jampens ${ }^{8}$, M. Tobin ${ }^{4}$, L. Tomassetti2 ${ }^{21, f}$, X. Tong ${ }^{5}$, D. Torres Machado ${ }^{1}$, D.Y. Tou ${ }^{13}$, E. Trifonova ${ }^{83}$, C. Trippl ${ }^{49}$, G. Tuci ${ }^{6}$, A. Tully ${ }^{49}$, N. Tuning ${ }^{32,48}$, A. Ukleja ${ }^{36}$, D.J. Unverzagt ${ }^{17}$, E. Ursov ${ }^{83}$, A. Usachov ${ }^{32}$, A. Ustyuzhanin ${ }^{42,82}$, U. Uwer ${ }^{17}$, A. Vagner ${ }^{84}$, V. Vagnoni ${ }^{20}$, A. Valassi ${ }^{48}$, G. Valenti ${ }^{20}$, N. Valls Canudas ${ }^{85}$, M. van Beuzekom ${ }^{32}$, M. Van Dijk ${ }^{49}$, H. Van Hecke ${ }^{67}$, E. van Herwijnen ${ }^{83}$, C.B. Van Hulse ${ }^{18}$, M. van Veghel ${ }^{79}$, R. Vazquez Gomez ${ }^{45}$, P. Vazquez Regueiro ${ }^{46}$, C. Vázquez Sierra ${ }^{48}$, S. Vecchi ${ }^{21}$, J.J. Velthuis ${ }^{54}$, M. Veltri ${ }^{22, r}$, A. Venkateswaran ${ }^{68}$, M. Veronesi ${ }^{32}$, M. Vesterinen ${ }^{56}$, D. Vieira ${ }^{65}$, M. Vieites Diaz ${ }^{49}$, H. Viemann ${ }^{76}$, X. Vilasis-Cardona ${ }^{85}$, E. Vilella Figueras ${ }^{60}$, A. Villa ${ }^{20}$, P. Vincent ${ }^{13}$, F.C. Volle ${ }^{11}$,

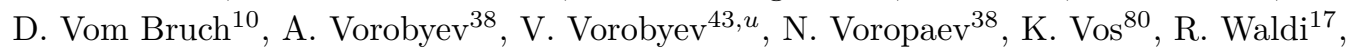

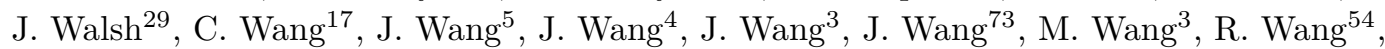
Y. Wang ${ }^{7}$, Z. Wang ${ }^{50}$, Z. Wang ${ }^{3}$, Z. Wang ${ }^{6}$, J.A. Ward ${ }^{56}$, N.K. Watson ${ }^{53}$, S.G. Weber ${ }^{13}$, D. Websdale ${ }^{61}$, C. Weisser ${ }^{64}$, B.D.C. Westhenry ${ }^{54}$, D.J. White ${ }^{62}$, M. Whitehead ${ }^{54}$, A.R. Wiederhold ${ }^{56}$, D. Wiedner ${ }^{15}$, G. Wilkinson ${ }^{63}$, M. Wilkinson ${ }^{68}$, I. Williams ${ }^{55}$, M. Williams ${ }^{64}$, M.R.J. Williams ${ }^{58}$, F.F. Wilson ${ }^{57}$, W. Wislicki ${ }^{36}$, M. Witek ${ }^{35}$, L. Witola ${ }^{17}$, G. Wormser ${ }^{11}$, S.A. Wotton ${ }^{55}$, H. $\mathrm{Wu}^{68}$, K. Wyllie ${ }^{48}$, Z. Xiang ${ }^{6}$, D. Xiao ${ }^{7}$, Y. Xie ${ }^{7}$, A. $\mathrm{Xu}^{5}$, J. Xu ${ }^{6}, \mathrm{~L} \mathrm{Xu}^{3}$,

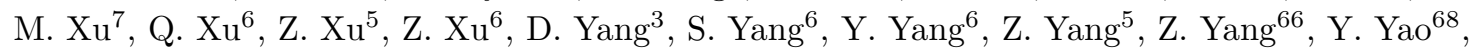
L.E. Yeomans ${ }^{60}$, H. Yin ${ }^{7}$, J. Yu ${ }^{71}$, X. Yuan ${ }^{68}$, O. Yushchenko ${ }^{44}$, E. Zaffaroni ${ }^{49}$, M. Zavertyaev ${ }^{16, t}$, M. Zdybal ${ }^{35}$, O. Zenaiev ${ }^{48}$, M. Zeng ${ }^{3}$, D. Zhang ${ }^{7}$, L. Zhang ${ }^{3}$, S. Zhang ${ }^{71}$, S. Zhang ${ }^{5}$, Y. Zhang ${ }^{5}$, Y. Zhang ${ }^{63}$, A. Zharkova ${ }^{83}$, A. Zhelezov ${ }^{17}$, Y. Zheng ${ }^{6}$, T. Zhou ${ }^{5}$, X. Zhou ${ }^{6}$, Y. Zhou ${ }^{6}$, V. Zhovkovska ${ }^{11}$, X. Zhu ${ }^{3}$, X. Zhu ${ }^{7}$, Z. Zhu ${ }^{6}$, V. Zhukov ${ }^{14,40}$, J.B. Zonneveld ${ }^{58}$, Q. Zou ${ }^{4}$, S. Zucchelli ${ }^{20, d}$, D. Zuliani ${ }^{28}$ and G. Zunica ${ }^{62}$

1 Centro Brasileiro de Pesquisas Físicas (CBPF), Rio de Janeiro, Brazil

2 Universidade Federal do Rio de Janeiro (UFRJ), Rio de Janeiro, Brazil

3 Center for High Energy Physics, Tsinghua University, Beijing, China

4 Institute Of High Energy Physics (IHEP), Beijing, China

5 School of Physics State Key Laboratory of Nuclear Physics and Technology, Peking University, Beijing, China

6 University of Chinese Academy of Sciences, Beijing, China

7 Institute of Particle Physics, Central China Normal University, Wuhan, Hubei, China

8 Univ. Savoie Mont Blanc, CNRS, IN2P3-LAPP, Annecy, France

9 Université Clermont Auvergne, CNRS/IN2P3, LPC, Clermont-Ferrand, France

10 Aix Marseille Univ, CNRS/IN2P3, CPPM, Marseille, France

11 Université Paris-Saclay, CNRS/IN2P3, IJCLab, Orsay, France

12 Laboratoire Leprince-Ringuet, CNRS/IN2P3, Ecole Polytechnique, Institut Polytechnique de Paris, Palaiseau, France

13 LPNHE, Sorbonne Université, Paris Diderot Sorbonne Paris Cité, CNRS/IN2P3, Paris, France

14 I. Physikalisches Institut, RWTH Aachen University, Aachen, Germany

15 Fakultät Physik, Technische Universität Dortmund, Dortmund, Germany

16 Max-Planck-Institut für Kernphysik (MPIK), Heidelberg, Germany

17 Physikalisches Institut, Ruprecht-Karls-Universität Heidelberg, Heidelberg, Germany

18 School of Physics, University College Dublin, Dublin, Ireland

19 INFN Sezione di Bari, Bari, Italy

20 INFN Sezione di Bologna, Bologna, Italy

21 INFN Sezione di Ferrara, Ferrara, Italy

22 INFN Sezione di Firenze, Firenze, Italy

${ }^{23}$ INFN Laboratori Nazionali di Frascati, Frascati, Italy 
24 INFN Sezione di Genova, Genova, Italy

25 INFN Sezione di Milano, Milano, Italy

${ }^{26}$ INFN Sezione di Milano-Bicocca, Milano, Italy

27 INFN Sezione di Cagliari, Monserrato, Italy

28 Universita degli Studi di Padova, Universita e INFN, Padova, Padova, Italy

29 INFN Sezione di Pisa, Pisa, Italy

30 INFN Sezione di Roma La Sapienza, Roma, Italy

31 INFN Sezione di Roma Tor Vergata, Roma, Italy

32 Nikhef National Institute for Subatomic Physics, Amsterdam, Netherlands

33 Nikhef National Institute for Subatomic Physics and VU University Amsterdam, Amsterdam, Netherlands

34 AGH - University of Science and Technology, Faculty of Physics and Applied Computer Science, Kraków, Poland

35 Henryk Niewodniczanski Institute of Nuclear Physics Polish Academy of Sciences, Kraków, Poland

36 National Center for Nuclear Research (NCBJ), Warsaw, Poland

37 Horia Hulubei National Institute of Physics and Nuclear Engineering, Bucharest-Magurele, Romania

38 Petersburg Nuclear Physics Institute NRC Kurchatov Institute (PNPI NRC KI), Gatchina, Russia

39 Institute for Nuclear Research of the Russian Academy of Sciences (INR RAS), Moscow, Russia

40 Institute of Nuclear Physics, Moscow State University (SINP MSU), Moscow, Russia

41 Institute of Theoretical and Experimental Physics NRC Kurchatov Institute (ITEP NRC KI), Moscow, Russia

42 Yandex School of Data Analysis, Moscow, Russia

43 Budker Institute of Nuclear Physics (SB RAS), Novosibirsk, Russia

44 Institute for High Energy Physics NRC Kurchatov Institute (IHEP NRC KI), Protvino, Russia, Protvino, Russia

45 ICCUB, Universitat de Barcelona, Barcelona, Spain

46 Instituto Galego de Física de Altas Enerxías (IGFAE), Universidade de Santiago de Compostela, Santiago de Compostela, Spain

47 Instituto de Fisica Corpuscular, Centro Mixto Universidad de Valencia - CSIC, Valencia, Spain

48 European Organization for Nuclear Research (CERN), Geneva, Switzerland

49 Institute of Physics, Ecole Polytechnique Fédérale de Lausanne (EPFL), Lausanne, Switzerland

50 Physik-Institut, Universität Zürich, Zürich, Switzerland

51 NSC Kharkiv Institute of Physics and Technology (NSC KIPT), Kharkiv, Ukraine

52 Institute for Nuclear Research of the National Academy of Sciences (KINR), Kyiv, Ukraine

53 University of Birmingham, Birmingham, United Kingdom

54 H.H. Wills Physics Laboratory, University of Bristol, Bristol, United Kingdom

55 Cavendish Laboratory, University of Cambridge, Cambridge, United Kingdom

56 Department of Physics, University of Warwick, Coventry, United Kingdom

57 STFC Rutherford Appleton Laboratory, Didcot, United Kingdom

58 School of Physics and Astronomy, University of Edinburgh, Edinburgh, United Kingdom

59 School of Physics and Astronomy, University of Glasgow, Glasgow, United Kingdom

60 Oliver Lodge Laboratory, University of Liverpool, Liverpool, United Kingdom

61 Imperial College London, London, United Kingdom

62 Department of Physics and Astronomy, University of Manchester, Manchester, United Kingdom

63 Department of Physics, University of Oxford, Oxford, United Kingdom

64 Massachusetts Institute of Technology, Cambridge, MA, United States

65 University of Cincinnati, Cincinnati, OH, United States

66 University of Maryland, College Park, MD, United States

67 Los Alamos National Laboratory (LANL), Los Alamos, United States

68 Syracuse University, Syracuse, NY, United States

69 School of Physics and Astronomy, Monash University, Melbourne, Australia, associated to ${ }^{56}$ 
Pontifícia Universidade Católica do Rio de Janeiro (PUC-Rio), Rio de Janeiro, Brazil, associated to ${ }^{2}$

71 Physics and Micro Electronic College, Hunan University, Changsha City, China, associated to ${ }^{7}$

72 Guangdong Provincial Key Laboratory of Nuclear Science, Guangdong-Hong Kong Joint Laboratory of Quantum Matter, Institute of Quantum Matter, South China Normal University, Guangzhou, China, associated to ${ }^{3}$

73 School of Physics and Technology, Wuhan University, Wuhan, China, associated to ${ }^{3}$

74 Departamento de Fisica, Universidad Nacional de Colombia, Bogota, Colombia, associated to ${ }^{13}$

75 Universität Bonn - Helmholtz-Institut für Strahlen und Kernphysik, Bonn, Germany, associated to 17

76 Institut für Physik, Universität Rostock, Rostock, Germany, associated to ${ }^{17}$

77 Eotvos Lorand University, Budapest, Hungary, associated to ${ }^{48}$

78 INFN Sezione di Perugia, Perugia, Italy, associated to ${ }^{21}$

79 Van Swinderen Institute, University of Groningen, Groningen, Netherlands, associated to ${ }^{32}$

80 Universiteit Maastricht, Maastricht, Netherlands, associated to ${ }^{32}$

81 National Research Centre Kurchatov Institute, Moscow, Russia, associated to ${ }^{41}$

82 National Research University Higher School of Economics, Moscow, Russia, associated to ${ }^{42}$

83 National University of Science and Technology "MISIS", Moscow, Russia, associated to ${ }^{41}$

84 National Research Tomsk Polytechnic University, Tomsk, Russia, associated to ${ }^{41}$

${ }^{85}$ DS4DS, La Salle, Universitat Ramon Llull, Barcelona, Spain, associated to 45

86 Department of Physics and Astronomy, Uppsala University, Uppsala, Sweden, associated to ${ }^{59}$

87 University of Michigan, Ann Arbor, United States, associated to ${ }^{68}$

a Universidade Federal do Triângulo Mineiro (UFTM), Uberaba-MG, Brazil

${ }^{b}$ Hangzhou Institute for Advanced Study, UCAS, Hangzhou, China

c Università di Bari, Bari, Italy

d Università di Bologna, Bologna, Italy

e Università di Cagliari, Cagliari, Italy

$f$ Università di Ferrara, Ferrara, Italy

$g$ Università di Firenze, Firenze, Italy

$h$ Università di Genova, Genova, Italy

${ }^{i}$ Università degli Studi di Milano, Milano, Italy

j Università di Milano Bicocca, Milano, Italy

k Università di Modena e Reggio Emilia, Modena, Italy

${ }^{l}$ Università di Padova, Padova, Italy

$m$ Scuola Normale Superiore, Pisa, Italy

$n$ Università di Pisa, Pisa, Italy

- Università della Basilicata, Potenza, Italy

p Università di Roma Tor Vergata, Roma, Italy

$q$ Università di Siena, Siena, Italy

$r$ Università di Urbino, Urbino, Italy

$s$ MSU - Iligan Institute of Technology (MSU-IIT), Iligan, Philippines

${ }^{t}$ P.N. Lebedev Physical Institute, Russian Academy of Science (LPI RAS), Moscow, Russia

u Novosibirsk State University, Novosibirsk, Russia

$\dagger$ Deceased 